\title{
Grasp Analysis Tools for Synergistic Underactuated Robotic Hands
}

\author{
Marco Gabiccini $^{1,2,4}$, Edoardo Farnioli ${ }^{1,4}$, Antonio Bicchi ${ }^{1,3,4}$ \\ ${ }^{1}$ Research Center “E. Piaggio”, Università di Pisa, Largo Lucio Lazzarino 1, 56122 Pisa, Italy \\ ${ }^{2}$ Department of Civil and Industrial Engineering, Università di Pisa, Largo Lucio Lazzarino 1, 56122 \\ Pisa, Italy \\ ${ }^{3}$ Department of Information Engineering, Università di Pisa, Largo Lucio Lazzarino 1, 56122 Pisa, Italy \\ ${ }^{4}$ Department of Advanced Robotics, Istituto Italiano di Tecnologia, Via Morego 30, 16163 Genova, Italy \\ E-mail: \{marco.gabiccini, edoardo.farnioli, antonio.bicchi\}@iit.it
}

Keywords: grasp analysis, compliant grasps, underactuated robotic hands, soft synergies, structural properties, grasp taxonomy.

\begin{abstract}
Despite being a classical topic in robotics, the research on dexterous robotic hands still stirs a lively research activity. The current interest is especially attracted by underactuated robotic hands where a high number of degrees of freedom (DoFs), and a relatively low number of degrees of actuation (DoAs) coexist. The correlation between the DoFs obtained through a wise distribution of actuators is aimed at simplifying the control with a minimal loss of dexterity. In this sense, the application of bio-inspired principles is bringing toward a more conscious design. This work proposes new, general approaches for the analysis of grasps with synergistic ${ }^{1}$ underactuated robotic hands.

After a review of the quasi-static equations describing the system, where also contact preload is considered, two different approaches to the analysis are presented. The first one is based on a systematic combination of the equations. The independent and the dependent variables are defined, and cause-effect relationships between them are found. In addition, remarkable properties of the grasp, as the subspace of controllable internal force and the grasp compliance, are worked out in symbolic form. Then, some relevant kinds of tasks, as pure squeeze, spurious squeeze and kinematic grasp displacements, are defined, in terms of nullity or non-nullity of proper variables. The second method of analysis shows how to discover the feasibility of the pre-defined tasks, operating a systematic decomposition of the solution space of the system. As a result, the inputs to be given to the hand, in order to achieve the desired system displacements, are found. The study of the feasible variations is carried out arriving to discover all the combinations of nullity and/or non-nullity variables which are allowed by the equations describing the system. Numerical results are presented both for precision and power grasps, finding forces and displacements that the hand can impose to the object, and showing which properties are preserved after the introduction of a synergistic underactuation mechanism.

\section{INTRODUCTION}

The design of robotic hands was directed for long time to obtain a better dexterity and capability in manipulation tasks. This goal was sought by increasing complexity and number of degrees of freedom, with remarkable examples of robotic hand designs following this trend represented by the \footnotetext{
analysis is performed, ranging from the common divergent inputs to multiple neurons at the neural level, the activity of concurrently active motor units at the muscle level, to covariation patterns among digit joints during reach-to-grasp and manipulation tasks at the kinematic level [Santello et al., 2013]. In the present paper, as usually common in the robotics community, we will adopt the kinematic level definition of synergies, e.g., finger joint covariation patterns in grasping and manipulation tasks.
}

${ }^{1}$ The term synergies in human hand control studies refers to various phenomena and in ways that reflect the level at which the
\end{abstract}


UTAH/MIT hand [Jacobsen et al., 1984], the Robonaut Hand [Lovchik and Diftler, 1999], the Shadow hand [Shadow Robot Company Ltd., 2009] and the DLR hand arm system [Grebenstein et al., 2012]. One of the main issues of this approach is that a large number of degrees of freedom implies many actuators, with a consequent growth of size, weight and cost. With such kind of devices, another disadvantage is the difficulty in control. Finding a joint control law, able to perform a given task, may become a difficult job, mainly due to the complex kinematic structures of the hands. Starting from these observations, different kinds of underactuation strategies were introduced and studied [Bicchi, 2000, Birglen et al., 2008]. In this direction, a recently considered approach is to take inspiration from the human hand, not just from a biomechanic point of view, but also for the control aspects.

Despite significant differences in the definitions and in the requirements of the investigated tasks, many neuroscience studies such as [Fish and Soechting, 1992], [Angelaki and Soechting, 1993], [Soechting and Flanders, 1997], [Santello et al., 1998], [Latash et al., 2005], [Thakur et al., 2008] and [Castellini and van der Smagt, 2013] to mention only a few, share a main observation: simultaneous motion of multiple digits occurs in a consistent fashion, even when the task may require a fairly high degree of movement individuation such as grasping a small object or typing.

One of the main quantitative results is that a large variety of common grasps that humans can do is well described by just five synergies, being the first two important enough to explain $80 \%$ of the variance in grasp postures. This suggests the possibility to change the basis of the description, from the joint space to the human-inspired postural synergy space. In [Ciocarlie et al., 2007] this idea was implemented via software to control the hand for grasp planning, interrupting the rigid coordination of a finger when it arrives in contact with the object to be grasped. A second way, proposed in [Brown and Asada, 2007], follows the road of an hardware implementation, where two kinds of synergies can be achieved by changing the set of actuation pulleys. In this case, when a first contact with the object achieved, either stable or not, the hand has to stop its motion. Differently from the previous one, the soft synergies approach [Gabiccini et al., 2011] avoids the problems due to a strict correlation between joint motions. In this case the synergistic movement is imposed to a virtual hand, attracting the real one via a generalized spring. The influence of synergies in motion and forces is investigated in [Prattichizzo et al., 2011]. The contact force optimization problem was investigated in [Gabiccini et al., 2011], taking into account the limitation imposed in the controllability by the underactation. Recently, the possibility to obtain a soft synergy like controllability using adaptive synergies was studied in [Grioli et al., 2012, Catalano et al., 2012], in order to achieve both the results to get human-inspired movement and an easier mechanical implementation.

This paper, based in part on [Gabiccini et al., 2012], deals with the quasi-static analysis of synergistic underactuated grasps, and proposes an approach where the set of equations describing the system is treated as a whole. Results are not limited to provide the controllable system variations by synergistic actuation. In addition to this, systematic ways to discover relevant properties of the grasp are provided, even in symbolic form, and a method to define and study the feasibility of some tasks is given. In Section 2 a review of the analytical description of the problem, based on the screw theory [Murray et al., 1994, Davidson and Hunt, 2004], is presented. Since also the derivative terms of the Jacobian matrix are taken into account, configurations with contact force preload can be rigorously analysed by the proposed method. Our choice is to use an object-centric formulation, obtaining a constant grasp matrix and some simplifications to describe rigid object displacements. The resulting system of equations is treated with two different approaches. The first, in Section 3, presents a systematic way to elaborate the system of equations. Input and output variables are defined and used in an algorithm of Gauss-Jordan elimination, extended to act on block partitioned matrices. In consequence of this, the relationships between the controls and the consequent hand/object configurations are given, both for displacements and forces, in two different ways, one more effective for numerical applications, the other more appropriate with symbolic forms of the equations. This leads to find relevant properties of the grasp, e.g. a basis for the controllable internal forces, controllable object displacements and the grasp 
compliance, discussed in Section 4. In Sections 5 and 6, the main categories of tasks are defined in terms of nullity or not-nullity of some variables. These definitions can be used to suitably apply the reduced row echelon form (RREF) to the solution space of the system, ascertaining their feasibility and the necessary inputs to be applied in order to perform them. The idea to distinguish different kinds of solutions is expanded in Section 7, where the equations describing the kineto-static behavior of the system are analyzed to discover which combinations of nullity or non-nullity are allowed, until a complete classification of the solution space is obtained. In Section 8 some words are spent to describe how the methods proposed in the paper could be applied to the study of robotic hands characterized by other types of (under-)actuation. To conclude, in Section 9, numerical examples are presented for tip and power grasps. Both cases are firstly studied as if the hand were completely actuated, finding out the hand manipulation capabilities. Then, the synergistic underactuation is introduced verifying which possibilities are lost and which other still persist.

\section{SYSTEM DESCRIPTION}

A basic assumption in the paper is that all bodies are modelled as rigid. Thanks to this, it is possible to use the wide variety of mathematical tools developed to describe rigid body motions. However, the presence of compliance in the system is allowed in a lumped way. For example, as we will further discuss later, the softness of the contact fingertip and/or the grasped object can be described by introducing contact virtual springs.

It is worth observing that, as usually done in literature, we separate the investigation of the system capabilities, e.g. the contact forces on the object actually controllable by the hand, to the restriction imposed by the friction limits, assuming that a subsequent classical force optimization procedure can lead to a feasible solution.

With reference to Figure 1, we model a hand as a collection of serial robots manipulating an object. An inertial frame $\{A\}=\left\{O_{a} ; x_{a}, y_{a}, z_{a}\right\}$ is attached to the palm. On the $i^{\text {th }}$ of the $p$ contact points we place a frame $\left\{C_{i}^{h}\right\}=\left\{O_{c_{i}^{h}} ; x_{c_{i}^{h}}, y_{c_{i}^{h}}, z_{c_{i}^{h}}\right\}$ attached to the finger link, and a frame $\left\{C_{i}^{o}\right\}=\left\{O_{c_{i}^{o}} ; x_{c_{i}^{o}}, y_{c_{i}^{o}}, z_{c_{i}^{o}}\right\}$ attached to the object. A reference frame $\{B\}=\left\{O_{b} ; x_{b}, y_{b}, z_{b}\right\}$ is fixed on the grasped object: later

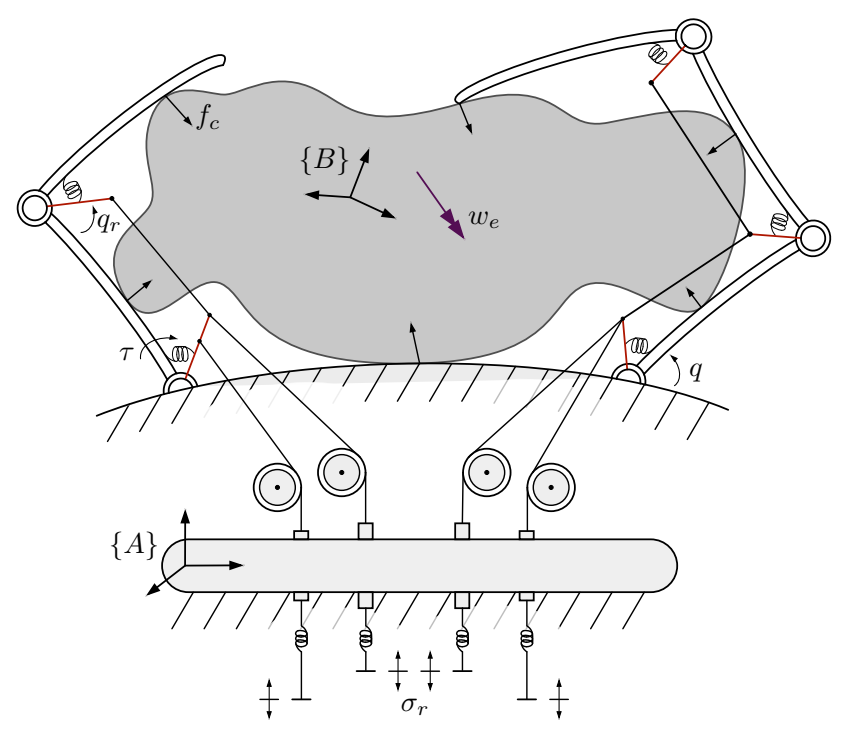

Figure 1: Compliant grasp by an underactuated robotic hand. 
in the text we will refer to it as the body frame. With the symbols $\xi_{x y}^{z}$ we indicate the twist of the frame $\{Y\}$ w.r.t. $\{X\}$, as described by an observer attached to $\{Z\}$. Denoting with $g_{t z} \in S E(3)$ the posture of frame $\{Z\}$ with respect to $\{T\}$, according to [Murray et al., 1994], we define the adjoint matrix, $\operatorname{Ad}_{g} \in \mathbb{R}^{6 \times 6}: s e(3) \rightarrow s e(3)$, as an operator able to transform twists by the law

$$
\xi_{x y}^{t}=\operatorname{Ad}_{g_{t z}} \xi_{x y}^{z} .
$$

Indicating with $w_{x}^{y}$ the wrench that the frame $\{X\}$ exerts on the frame $\{Y\}$, and in $\{Y\}$ components, we introduce the co-adjoint matrix operator, $\operatorname{Ad}_{g}^{-T} \in \mathbb{R}^{6 \times 6}: s e^{*}(3) \rightarrow s e^{*}(3)$, transforming wrenches according to the law

$$
w_{x}^{t}=\operatorname{Ad}_{g_{t y}}^{-T} w_{x}^{y}
$$

For reader convenience, the explicit form of the homogeneous and adjoint operators is given in Appendix

\begin{tabular}{|c|c|}
\hline Notation & Definition \\
\hline$\delta \nu$ & variation of variable $\nu$ \\
\hline $\bar{\nu}$ & value of variable $\nu$ in reference configuration \\
\hline$\sharp \nu$ & dimensions of vector $\nu$ \\
\hline$\{A\}$ & palm (inertial) frame \\
\hline$\left\{C_{i}^{h}\right\}$ & $i^{\text {th }}$ contact frame attached to the hand \\
\hline$\left\{C_{i}^{o}\right\}$ & $i^{\text {th }}$ contact frame attached to the object \\
\hline$\{B\}$ & object frame \\
\hline$q \in \mathbb{R}^{\sharp q}$ & joint variables \\
\hline$q_{r} \in \mathbb{R}^{\sharp q}$ & joint reference variables \\
\hline$\tau \in \mathbb{R}^{\sharp q}$ & joint torques \\
\hline$\sigma \in \mathbb{R}^{\sharp \sigma}$ & synergy variables \\
\hline$\sigma_{r} \in \mathbb{R}^{\sharp \sigma}$ & synergy reference variables \\
\hline$\eta \in \mathbb{R}^{\sharp \sigma}$ & synergy generalized forces \\
\hline$p$ & number of contact points \\
\hline & number of hand/object interaction constraints \\
\hline$u \in \mathbb{R}^{6}$ & position and orientation of the object frame \\
\hline$\xi_{x y}^{z} \in \mathbb{R}^{6}$ & $\begin{array}{l}\text { in the motion of the frame }\{Y\} \text { relative to }\{X\} \text {, it } \\
\text { represents the twist of the frame }\{Z\} \text { as attached to }\{Y\} \text {, } \\
\text { with components expressed in }\{Z\}\end{array}$ \\
\hline$f_{c_{i}^{h}}^{c_{i}^{o}}$ & contact force/torque exerted from $\left\{C_{i}^{h}\right\}$ to $\left\{C_{i}^{o}\right\}$ with components \\
\hline$w_{x}^{y} \in \mathbb{R}^{6}$ & $\begin{array}{l}\text { and moments relative to the frame }\left\{C_{i}^{o}\right\} \\
\text { wrench exerted by }\{X\} \text { on }\{Y\} \text {, with components } \\
\text { and moments relative to }\{Y\}\end{array}$ \\
\hline${ }^{a_{J}} \in \mathbb{R}^{c \times \sharp q}$ & hand Jacobian matrix in inertial frame \\
\hline$c^{o} J \in \mathbb{R}^{c \times \sharp q}$ & hand Jacobian matrix in object contact frames \\
\hline$S \in \mathbb{R}^{\sharp q \times \sharp \sigma}$ & synergy matrix \\
\hline${ }^{b} G \in \mathbb{R}^{6 \times c}$ & grasp matrix in body frame \\
\hline
\end{tabular}
A. Other definitions and the notation used in the text are summarized in Table 1.

Table 1: Notation for grasp analysis.

\subsection{Equilibrium Equation of the Object}

Let $w_{e}^{b} \in \mathbb{R}^{6}$ be the external wrench acting on the object with components expressed in $\{B\}$. Similarly, we indicate with $w_{c_{i}^{h}}^{c_{i}^{o}}$ the wrench that the hand exerts on the $i^{\text {th }}$ contact point on the object, 
with components in $\left\{C_{i}^{o}\right\}$. The equilibrium of the frame $\{B\}$ requires that the sum of all force/moment contributions, with components in $\{B\}$, is null. This condition can be written as

$$
w_{e}^{b}+\sum_{i=1}^{p} \operatorname{Ad}_{g_{b c_{i}^{o}}}^{-T} w_{c_{i}^{h}}^{c_{i}^{o}}=0 .
$$

The hand/object interaction can be described as a six-dimensional constraint for the position and orientation of the local contact frame. Usually, however, depending on the nature of the contact type, the constrained directions are less than six. In this way, we assume to describe the contact interaction with a vector $f_{c_{i}^{h}}^{c_{i}^{o}} \in \mathbb{R}^{c_{i}}$, with $c_{i} \leq 6$, that takes into account only the directions constrained. More details will be provided in Section 2.5. For each contact point we introduce a matrix $B_{i} \in \mathbb{R}^{6 \times c_{i}}$ able to map the local interaction in a complete wrench as follows

$$
w_{c_{i}^{h}}^{c_{i}^{o}}=B_{i} f_{c_{i}^{h}}^{c_{i}^{o}}
$$

Substituting (4) in (3), we obtain

$$
w_{e}^{b}+\sum_{i=1}^{p} \operatorname{Ad}_{g_{b c_{i}^{o}}}^{-T} B_{i} f_{c_{i}^{h}}^{c_{i}^{o}}=0
$$

Defining $c=\sum_{i=1}^{p} c_{i}$ as the total number of contact constrain acting on the object, we define the grasp matrix in body frame, ${ }^{b} G \in \mathbb{R}^{6 \times c}$, as

$$
{ }^{b} G=\left[\begin{array}{llllll}
\operatorname{Ad}_{g_{b c_{1}^{o}}}^{-T} B_{1} & \cdots & \operatorname{Ad}_{g_{b c_{i}^{o}}}^{-T} B_{i} & \cdots & \operatorname{Ad}_{g_{b c_{p}^{o}}}^{-T} B_{p}
\end{array}\right] .
$$

Equation (6) allows us to express the object equilibrium equation in the form

$$
w_{e}^{b}+{ }^{b} G f_{c^{h}}^{c^{o}}=0
$$

where $f_{c^{h}}^{c^{o}}=\left[f_{c_{1}^{h}}^{c_{1}^{o T}}, \ldots, f_{c_{p}^{h}}^{c_{p}^{o T}}\right]^{T} \in \mathbb{R}^{c}$ is a vector collecting all the actions performed by the hand on the object. It is important to underline that the grasp matrix in body frame is a constant matrix if the object contact points do not change. In consequence of this, to find a law relating small perturbations of the variables involved, we can calculate the differential of (7). It follows that the perturbed equilibrium equation can be written as

$$
\delta w_{e}^{b}+{ }^{b} G \delta f_{c^{h}}^{c^{o}}=0 .
$$

\subsection{Congruence Equation of the Object}

The twist of the $i^{\text {th }}$ contact frame $\left\{C_{i}^{o}\right\}$ on the object can be described as a function of the twist of the object frame $\{B\}$. To do this we use the adjoint matrix, presented in (1), as follows

$$
\xi_{a b}^{c_{i}^{o}}=\operatorname{Ad}_{g_{c_{i}^{o b}}} \xi_{a b}^{b} .
$$

As briefly discussed before, usually the contact constraints do not involve all the displacement directions. As explained in [Bicchi, 1994], the matrix $B_{i}^{T}$ is able to select the terms of velocity violating the contact constraints, as well as the matrix $B_{i}$ in (4) can expand the force/moment vector in a complete wrench. Introducing the vector $v_{a b}^{c_{i}^{o}} \in \mathbb{R}^{c_{i}}$, containing only the terms of the $i^{\text {th }}$ velocity violating constraints, the equation relating the body frame twist and the velocity of the $i^{\text {th }}$ contact point frame can be expressed as

$$
v_{a b}^{c_{i}^{o}}=B_{i}^{T} \xi_{a b}^{c_{i}^{o}}=B_{i}^{T} \operatorname{Ad}_{g_{c_{i}^{o} b}} \xi_{a b}^{b} .
$$


Defining $v_{a b}^{c^{o}}=\left[\begin{array}{lllll}v_{a b}^{c_{1}^{o}} & \cdots & v_{a b}^{c_{i}^{o} T} & \cdots & v_{a b}^{c_{p}^{o}}\end{array}\right]^{T} \in \mathbb{R}^{c}$, using (9) and (10), we can write

$$
v_{a b}^{c^{o}}=\left[\left(B_{1}^{T} \operatorname{Ad}_{g_{1} b}\right)^{T} \cdots\left(B_{i}^{T} \operatorname{Ad}_{g_{c_{i}^{o} b}}\right)^{T} \cdots\left(B_{p}^{T} \operatorname{Ad}_{g_{c_{p} b}}\right)^{T}\right]^{T} \xi_{a b}^{b} .
$$

By a direct comparison between (11) and the definition of the grasp matrix in (6), immediately follows that

$$
v_{a b}^{c^{o}}={ }^{b} G^{T} \xi_{a b}^{b} .
$$

Remembering that the rotational part of the term $\xi_{a b}^{b} d t$ does not represent a variation of coordinates, we have to introduce a vector $u \in \mathbb{R}^{6}$ able to parametrize $S E(3)$. A possible approach is to choose a parametrization vector composed by the same translational part of $\xi_{a b}^{b}$ and Euler's angles in the rotational part, as described in [Sciavicco and Siciliano, 2000]. A convenient approach is to introduce a virtual kinematic chain, describing the object configuration with respect to the absolute frame. In this case the object Jacobian matrix, that is the Jacobian of the virtual chain, can be used to parametrize the object twist, as follows

$$
\xi_{a b}^{b}=J_{o}(u) \dot{u} .
$$

Defining the variation of the contact frame of the object as $\delta C_{a b}^{c^{o}}=v_{a b}^{c^{o}} d t$, we can substitute (13) in (12). Introducing the matrix ${ }^{b} G_{t}^{T}(u)={ }^{b} G^{T} J_{o}(u)$, we can write the congruence equation of the object as

$$
\delta C_{a c^{o}}^{c^{o}}={ }^{b} G_{t}^{T}(u) \delta u .
$$

\subsection{Congruence Equation of the Hand}

Letting the vector $\xi_{a c_{i}^{h}}^{a} \in \mathbb{R}^{6}$ be the twist of the frame $\left\{C_{i}^{h}\right\}$ with respect to $\{A\}$, with components expressed in $\{A\}$, as described in [Murray et al., 1994] and summarized in Appendix B, we introduce the spatial Jacobian matrix ${ }^{a} J_{i}\left(q_{i}\right) \in \mathbb{R}^{6 \times \sharp q_{i}}$, where $\sharp q_{i}$ is the number of joints that precedes the $i^{\text {th }}$ hand contact point in its own serial chain. This allows us to write the relationship

$$
\xi_{a c_{i}^{h}}^{a}={ }^{a} J_{i}\left(q_{i}\right) \dot{q}_{i}
$$

An object-centric description is obtained mapping (15) from the inertial frame $\{A\}$ to object frame $\left\{C_{i}^{o}\right\}$, thus using the adjoint operator as follows

$$
\xi_{a c_{i}^{h}}^{c_{i}^{o}}=\operatorname{Ad}_{g_{c_{i}^{o} a}(u)} \xi_{a c_{i}^{h}}^{a}=\operatorname{Ad}_{g_{c_{i}^{o} a}(u)} J_{i} J_{i}\left(q_{i}\right) \dot{q}_{i}
$$

The selection matrix $B_{i}^{T}$ can be used, as in (10), to maintain the terms of velocity in the directions violating contact constraints. This results in

$$
v_{a c_{i}^{h}}^{c_{i}^{o}}=B_{i}^{T} \operatorname{Ad}_{g_{c_{i}^{o} a}(u)^{a}} J_{i}\left(q_{i}\right) \dot{q}_{i}={ }^{c_{i}^{o}} J_{i}\left(q_{i}, u\right) \dot{q}_{i} .
$$

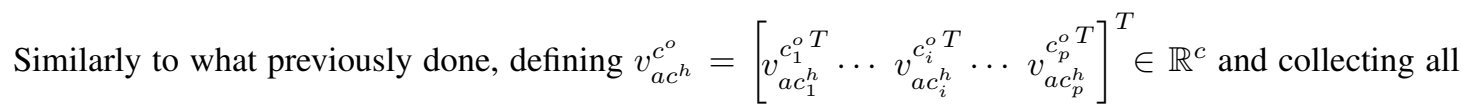
the joint parameters in the vector $q \in \mathbb{R}^{\sharp q}$, from (17) we can write

$$
v_{a c^{h}}^{c^{o}}={ }^{c^{o}} J(q, u) \dot{q}
$$

where ${ }^{c^{o}} J(q, u)$ is the hand Jacobian referred to the object contact frames. Multiplying (18) by $d t$, we find the congruence equation of the hand as

$$
\delta C_{a c^{h}}^{c^{o}}={ }^{c^{o} J}(q, u) \delta q .
$$




\subsection{Equilibrium Equation of the Hand}

From the kineto-static duality, coherently with the expression of the hand Jacobian, the map from the forces exerted by the hand $f_{c^{h}}^{c^{o}}$ to the joint torques $\tau \in \mathbb{R}^{\sharp q}$ is given by

$$
\tau={ }^{o} J^{T}(q, u) f_{c^{h}}^{c^{o}}
$$

Small perturbations of the system can be described by differentiation of (20), obtaining

$$
\delta \tau=\frac{\partial{ }^{c^{o} J^{T}}(q, u) f_{c^{h}}^{c^{o}}}{\partial q} \delta q+\frac{\partial{ }^{c^{o} J^{T}}(q, u) f_{c^{h}}^{c^{o}}}{\partial u} \delta u+\frac{\partial^{c^{o} J^{T}}(q, u) f_{c^{h}}^{c^{o}}}{\partial f} f_{c^{h}}^{c^{o}} .
$$

Defining the matrices

$$
Q=\frac{\partial^{c^{o} J^{T}}(q, u) f_{c^{h}}^{c^{o}}}{\partial q} \in \mathbb{R}^{\sharp q \times \sharp q}, \quad U=\frac{\partial^{c^{o} J^{T}(q, u) f_{c^{h}}^{c^{o}}}}{\partial u} \in \mathbb{R}^{\sharp q \times 6},
$$

the equation describing the perturbed equilibrium of the hand can be expressed as

$$
\delta \tau=Q \delta q+U \delta u+{ }^{c^{o}} J^{T} \delta f_{c^{h}}^{c^{o}} .
$$

Evaluating (23) in an equilibrium configuration, the terms $Q$ and $U$ become negligible if the initial contact forces are small or null. In Appendix $C$ we present a method to compute the derivatives in (22), based only on the knowledge of the elements composing (17), that is the spatial Jacobian and adjoint transformations, in an algorithmic way. From a practical point of view, this implies that symbolic calculations are not required to compute $(22)$.

\subsection{Hand/Object Interaction Model}

The contact between the hand and the object can be interpreted as a motion constraint. Typically, depending on the nature of materials involved, not all the directions of motions are limited. As an example, for the case of contact point without friction, only the direction normal to the contact surface is forbidden. This contact type is equivalent to having only one constraint, thus the dimension of the vector $f_{c_{i}^{o}}^{c_{i}^{o}}$ is $c_{i}=1$. Similarly, the contact point with friction, or hard finger, allows the presence of three components of force, on the contact, but no moment, $c_{i}=3$. As a last example we cite the soft finger case. With respect to the hard finger, it adds the possibility to transmit a moment around the normal vector to the contact surface, $c_{i}=4$.

In general, we are not justified to assume that the total number of motion constraints imposed by the interaction with the hand is equal to the degrees of freedom of the grasped object (six in 3D case, three in a planar case). On the contrary, we usually have more constraints that degrees of freedom, and this makes the problem hyperstatic, aka statically indeterminate. In these cases, the equilibrium equations are not sufficient to univocally determine the contact forces. To tackle this problem, we introduce a contact force model which is linear in the interference between the hand and the object. More specifically, for the $i^{\text {th }}$ contact point we introduce a stiffness matrix $K_{c_{i}} \in \mathbb{R}^{c_{i} \times c_{i}}$ containing the characteristics of the virtual contact spring. Accordingly, the $i^{\text {th }}$ force that the hand exerts on the object can be expressed as

$$
\delta f_{c_{i}^{h}}^{c_{i}^{o}}=K_{c_{i}}\left(\delta C_{a c_{i}^{h}}^{c_{o}^{o}}-\delta C_{a c_{i}^{o}}^{c_{i}^{o}}\right)
$$

Introducing $K_{c}=\operatorname{blkdiag}\left(K_{c_{1}}, \ldots, K_{c_{p}}\right) \in \mathbb{R}^{c \times c}$, as a block diagonal matrix, where the $i^{\text {th }}$ block element is $K_{c_{i}}$, the constitutive equation of the contact forces becomes

$$
\delta f_{c^{h}}^{c^{o}}=K_{c}\left(\delta C_{a c^{h}}^{c^{o}}-\delta C_{a c^{o}}^{c^{o}}\right)
$$




\subsection{Joint Actuation Model}

For the $i^{\text {th }}$ joint we introduce an elastic model relating the real configuration with a reference value, $q_{r_{i}} \in \mathbb{R}$, described by a torsional spring with constant stiffness $k_{q_{i}} \in \mathbb{R}$

$$
\tau_{i}=k_{q_{i}}\left(q_{r_{i}}-q_{i}\right)
$$

Defining $K_{q}=\operatorname{diag}\left(k_{q_{i}}\right) \in \mathbb{R}^{\sharp q \times \sharp q}$ as the matrix collecting all the joint stiffness, and introducing $q_{r} \in \mathbb{R}^{\sharp q}$ as the vector collecting the joint reference variables, by differentiation we can write the quasistatic actuation law as

$$
\delta \tau=K_{q}\left(\delta q_{r}-\delta q\right)
$$

\subsection{Underactuation of the Hand}

The equations presented up to here allows the description of a hand with elastic joints, but with an equal number of degrees of actuation and freedom. However, it is possible to extend the problem to include underactuated systems as well. To this end, we impose that the joint reference positions $q_{r}$ evolve on a manifold $\mathbb{S} \subseteq \mathbb{R}^{\sharp q}$ as a function of some postural synergy values $\sigma \in \mathbb{R}^{\sharp \sigma}$, where $\sharp \sigma \leq \sharp q$. Thus, in general we can write

$$
q_{r}=f(\sigma),
$$

where $f: \mathbb{R}^{\sharp \sigma} \rightarrow \mathbb{S} \subseteq \mathbb{R}^{\sharp q}$ is the synergy function. Calculating the differential of (28), and defining the synergy matrix as $S(\sigma)=\frac{\partial f(\sigma)}{\partial \sigma} \in \mathbb{R}^{\sharp q \times \sharp \sigma}$, the joint reference displacements are described as follows

$$
\delta q_{r}=S(\sigma) \delta \sigma
$$

Again, by virtue of the kineto-static duality, we can find that the vector $\eta \in \mathbb{R}^{\sharp \sigma}$ of the generalized forces at the synergy level is related to the joint torques by the relationship

$$
\eta=S^{T}(\sigma) \tau .
$$

Differentiating (30) and introducing the matrix $\Sigma(\sigma, \tau)=\frac{\partial S^{T}(\sigma) \tau}{\partial \sigma} \in \mathbb{R}^{\sharp \sigma \times \sharp \sigma}$, for the actuation forces we obtain

$$
\delta \eta=S^{T}(\sigma) \delta \tau+\Sigma(\sigma, \tau) \delta \sigma .
$$

For the sake of generality, we can introduce an elastic model even for the synergistic underactuation, similarly to what done before for the joints. Defining the synergy stiffness as the matrix $K_{\sigma}=\operatorname{diag}\left(k_{\sigma_{i}}\right) \in$ $\mathbb{R}^{\sharp \sigma \times \sharp \sigma}$, and introducing the vector of synergy references $\sigma_{r} \in \mathbb{R}^{\sharp \sigma}$, the actuation law appears as

$$
\delta \eta=K_{\sigma}\left(\delta \sigma_{r}-\delta \sigma\right)
$$

\subsection{The Fundamental Grasp Equation}

The set of equations composed collecting (8), (14), (19), (23), (25), (27), (29), (31) and (32) is able to describe a linear approximation of all the possible system displacements around an equilibrium 
configuration. The result is the Fundamental Grasp Equation (FGE) and it appears as

$$
\left[\begin{array}{ccccccccccc}
I_{\sharp f} & 0 & 0 & 0 & -K_{c} & K_{c} & 0 & 0 & 0 & 0 & 0 \\
-{ }^{c^{c}} \bar{J}^{T} & I_{\sharp \tau} & 0 & -\bar{U} & 0 & 0 & -\bar{Q} & 0 & 0 & 0 & 0 \\
0 & -\bar{S}^{T} & I_{\sharp \eta} & 0 & 0 & 0 & 0 & 0 & -\bar{\Sigma} & 0 & 0 \\
{ }^{b} G & 0 & 0 & 0 & 0 & 0 & 0 & 0 & 0 & I_{\sharp w} & 0 \\
0 & 0 & 0 & 0 & I_{\sharp C^{h}} & 0 & -c^{c^{o}} \bar{J} & 0 & 0 & 0 & 0 \\
0 & 0 & 0 & -{ }^{b} \bar{G}_{t}^{T} & 0 & I_{\sharp C^{o}} & 0 & 0 & 0 & 0 & 0 \\
0 & I_{\sharp \tau} & 0 & 0 & 0 & 0 & K_{q} & -K_{q} & 0 & 0 & 0 \\
0 & 0 & 0 & 0 & 0 & 0 & 0 & I_{\sharp q_{r}} & -\bar{S} & 0 & 0 \\
0 & 0 & I_{\sharp \sigma} & 0 & 0 & 0 & 0 & 0 & K_{\sigma} & 0 & -K_{\sigma}
\end{array}\right]\left[\begin{array}{c}
\delta f_{c^{h}}^{c^{o}} \\
\delta \tau \\
\delta \eta \\
\delta u \\
\delta C_{a c^{c}}^{c^{o}} \\
\delta C_{a c^{o}}^{c^{c}} \\
\delta q \\
\delta q_{r} \\
\delta \sigma \\
\delta w_{e}^{b} \\
\delta \sigma_{r}
\end{array}\right]=0
$$

where we indicated with $\bar{\nu}$ the value of the variable $\nu$ in the starting equilibrium point, and with the symbol $I_{\sharp \nu}$ an identity matrix of dimension $\sharp \nu$.

Equation (33) is a system of linear and homogeneous equations in the form $A \delta y=0$, where the coefficient matrix $A \in \mathbb{R}^{r_{a} \times c_{a}}$ is called Fundamental Grasp Matrix (FGM), and the vector $y \in \mathbb{R}^{c_{a}}$ collecting all the variable is the augmented configuration.

It is trivial to note that the constraint identified by (33) does not change its physical implications using a different order of both the equations and the variables. For reasons that will be clarified in Section 3.2.4, the chosen order of the equations and of the augmented configuration components derives from the specification to have square blocks on the main diagonal. However, it is always possible to obtain this characteristic from any other order of equations and variables, simply applying suitable permutation matrices.

\section{CONTROLLABLE SYSTEM PERTURBATIONS}

It is easy to find that the dimensions $r_{a}$ and $c_{a}$ of the Fundamental Grasp Matrix in (33) correspond to

$$
\begin{aligned}
& r_{a}=\sharp w+3 \sharp \tau+3 \sharp f+2 \sharp \sigma, \\
& c_{a}=2 \sharp w+3 \sharp \tau+3 \sharp f+3 \sharp \sigma .
\end{aligned}
$$

In the very general cases of interest, it $\operatorname{results} \operatorname{rank}(A)=r_{a}$, and we will assume it in the rest of the text. Exceptions are analytically possible but they refer to pathological situations of poor practical relevance.

All the solutions of (33) can be written by a basis for the nullspace of the Fundamental Grasp Matrix. We indicate this with the symbol $\Gamma \in \mathbb{R}^{r_{\gamma} \times c_{\gamma}}$. Taking into account (34), it immediately follows that

$$
\begin{aligned}
r_{\gamma} & =c_{a}, \\
c_{\gamma} & =c_{a}-r_{a}=\sharp w+\sharp \sigma .
\end{aligned}
$$

This implies that we can find an unique solution of (33) when it is known a number of independent variables, or inputs for the system, equal to $c_{\gamma}=\sharp w+\sharp \sigma$.

In continuity with the grasp analysis literature, we choose to consider the external wrench variation $\delta w_{e}^{b}$ as known or measurable. Moreover, we consider the synergy references as position-controlled. This corresponds to the practical case of an actuation by servo motors to control the synergy displacements $\delta \sigma_{r}$. Other choices are possible, maintaining constant $c_{\gamma}$, for example by substituting the external wrench $\delta w_{e}^{b}$ with the object displacement $\delta u$, or the reference synergy positions $\delta \sigma_{r}$ with synergy force actuation $\delta \eta$. The results of our analysis can be easily adapted to the above mentioned situations as well.

Next sections will show two different methods to obtain the complete configuration of the system as a function of the input variables. The first method, called Find-X, presented in Section 3.1, has the 
advantage of a simple and versatile application, very effective from a numerical point of view. On the contrary the second one, called GEROME-B, in Section 3.2, has the advantage to produce input/output equations with relevant physical sense, more effective when presented in symbolic form. Furthermore, it results an useful systematic method to merge some subspaces of interest in the grasp analysis, and we will better discuss these in Sections 4.2 and 4.3.

\section{$3.1 \quad$ Find-X Method}

From previous considerations, it follows that all the solution of (33) can be obtained as a combination of the columns of the solution space $\Gamma$. Thus, introducing a vector of parameters $x \in \mathbb{R}^{\sharp w+\sharp \sigma}$, every augmented configuration variation such that

$$
\delta y=\Gamma x
$$

will be a solution of the system.

As well as $\delta y$ is composed by a set of variables, we can divide the matrix $\Gamma$ in the corresponding portions as follows

$$
\left[\begin{array}{c}
\delta f_{c^{\circ}}^{c^{o}} \\
\delta \tau \\
\delta \eta \\
\delta u \\
\delta C_{a c^{c^{o}}}^{c^{h}} \\
\delta C_{a c^{o}}^{c^{o}} \\
\delta q \\
\delta q_{r} \\
\delta \sigma \\
\delta w_{e}^{b} \\
\delta \sigma_{r}
\end{array}\right]=\left[\begin{array}{l}
\Gamma_{f} \\
\Gamma_{\tau} \\
\Gamma_{\eta} \\
\Gamma_{u} \\
\Gamma_{C^{h}} \\
\Gamma_{C^{o}} \\
\Gamma_{q} \\
\Gamma_{q_{r}} \\
\Gamma_{\sigma} \\
\Gamma_{w} \\
\Gamma_{\sigma_{r}}
\end{array}\right] x
$$

where, for the block relative to the variable $\nu$, it results $\Gamma_{\nu} \in \mathbb{R}^{\sharp \nu \times c_{\gamma}}$.

Under the assumption that the inputs of the system are the external wrench and the synergy references, it is possible to calculate the vector $x$ in (37), responsible of the column combination of $\Gamma$, simply as

$$
x=\left[\begin{array}{c}
\Gamma_{w} \\
\Gamma_{\sigma_{r}}
\end{array}\right]^{-1}\left[\begin{array}{c}
\delta w_{e}^{b} \\
\delta \sigma_{r}
\end{array}\right] .
$$

The perturbed configuration of the system can be found by substituting (38) in (36) obtaining

$$
\delta y=\Gamma\left[\begin{array}{c}
\Gamma_{w} \\
\Gamma_{\sigma_{r}}
\end{array}\right]^{-1}\left[\begin{array}{c}
\delta w_{e}^{b} \\
\delta \sigma_{r}
\end{array}\right] .
$$

The invertibility of the matrix $\left[\begin{array}{cc}\Gamma_{w}^{T} & \Gamma_{\sigma_{r}}^{T}\end{array}\right]^{T}$ is acceptable with the same generality of the assumption that the coefficient matrix in (33) is full rank. In the eventuality of different inputs, it is immediate to find the corresponding portions of $\Gamma$ to calculate vector $x$ in the same way as in (38).

\subsection{GEROME-B: the Gauss Elementary Row Operation Method for Block Partitioned Matrix}

Since the system in (33) is linear and homogeneous, we can act on the coefficient matrix performing a Gauss-Jordan elimination. To do this maintaining the integrity of the sub-matrices composing the fundamental grasp matrix, we need to extend the Gauss Elementary Row Operations to process a block partitioned matrix. We can write the three Gauss elementary block operations as:

- exchanging the $i^{\text {th }}$ block row with the $j^{\text {th }}$ block row, 
- pre-multiplying the $i^{\text {th }}$ block row for a full column rank matrix $\Delta$,

- adding the $j^{\text {th }}$ block row, eventually multiplied for a suitable matrix $\Lambda$ to accord dimensions, to the $i^{\text {th }}$ block row.

As well as the original elimination method, we need to apply these rules without affecting the solution space of the system. To this aim, we remind the following

Theorem 1. Given the matrices $P \in \mathbb{R}^{r_{p} \times c_{p}}$ and $M \in \mathbb{R}^{r_{m} \times c_{m}}$, with $c_{m}=r_{p}$, it results $\mathcal{N}(M P)=$ $\mathcal{N}(P)$, if and only if $M$ is full column rank.

More details about this can be found in [Meyer, 2000]. In next sections, we will get three full rank matrices able to translate the Gauss elementary operations, as pre-multiplication matrices of a generic block partitioned matrix $P$, coefficient matrix of linear and homogeneous system of the type $P z=0$. Thanks to Theorem 1, we can guarantee the integrity of its own solution space.

\subsubsection{First Gauss Operation}

To translate the first rule, considering $P$ composed by $m$ block rows, we start from a partitioned identity matrix $I_{p} \in \mathbb{R}^{r_{p} \times r_{p}}$, such that the $i^{\text {th }}$ of $m$ blocks, $I_{i}$, has the same number of rows of the corresponding $i^{\text {th }}$ block row of $P, \forall i \in\{1, \ldots, m\}$. To obtain the effect of exchanging the $i^{\text {th }}$ and the $j^{\text {th }}$ block row of $P$, we have to pre-multiply it for a matrix in the form

$$
M_{i j}^{1}=\left[\begin{array}{ccccccccccccc}
I_{1} & \cdots & 0 & 0 & 0 & 0 & \cdots & 0 & 0 & 0 & 0 & \cdots & 0 \\
0 & \ddots & 0 & 0 & 0 & 0 & \cdots & 0 & 0 & 0 & 0 & . & 0 \\
0 & \cdots & I_{i-1} & 0 & 0 & 0 & \cdots & 0 & 0 & 0 & 0 & \cdots & 0 \\
0 & \cdots & 0 & 0 & 0 & 0 & \cdots & 0 & 0 & I_{j} & 0 & \cdots & 0 \\
0 & \cdots & 0 & 0 & I_{i+1} & 0 & \cdots & 0 & 0 & 0 & 0 & \cdots & 0 \\
0 & \cdots & 0 & 0 & 0 & \ddots & \cdots & 0 & 0 & 0 & 0 & \cdots & 0 \\
0 & \cdots & 0 & 0 & 0 & 0 & I & 0 & 0 & 0 & 0 & \cdots & 0 \\
0 & \cdots & 0 & 0 & 0 & 0 & \cdots & \ddots & 0 & 0 & 0 & \cdots & 0 \\
0 & \cdots & 0 & 0 & 0 & 0 & \cdots & 0 & I_{j-1} & 0 & 0 & \cdots & 0 \\
0 & \cdots & 0 & I_{i} & 0 & 0 & \cdots & 0 & 0 & 0 & 0 & \cdots & 0 \\
0 & \cdots & 0 & 0 & 0 & 0 & \cdots & 0 & 0 & 0 & I_{j+1} & \cdots & 0 \\
0 & . & 0 & 0 & 0 & 0 & \cdots & 0 & 0 & 0 & 0 & \ddots & 0 \\
0 & \cdots & 0 & 0 & 0 & 0 & \cdots & 0 & 0 & 0 & 0 & \cdots & I_{m}
\end{array}\right] .
$$

\subsubsection{Second Gauss Operation}

A matrix performing the second rule is obtained starting again from the partitioned identity matrix $I_{p}$, and substituting the $i^{\text {th }}$ identity block with a full rank matrix $\Delta$. The searched pre-multiplication matrix appears as

$$
M_{i i}^{2}(\Delta)=\left[\begin{array}{ccccccc}
I_{1} & \cdots & 0 & 0 & 0 & \cdots & 0 \\
0 & \ddots & 0 & 0 & 0 & \cdots & 0 \\
0 & \cdots & I_{i-1} & 0 & 0 & \cdots & 0 \\
0 & \cdots & 0 & \Delta & 0 & \cdots & 0 \\
0 & \cdots & 0 & 0 & I_{i+1} & \cdots & 0 \\
0 & \cdots & 0 & 0 & 0 & \ddots & 0 \\
0 & \cdots & 0 & 0 & 0 & \cdots & I_{m}
\end{array}\right]
$$




\subsubsection{Third Gauss Operation}

At last, to sum two rows, we place a matrix $\Lambda$, of appropriate dimensions, at the intersection of the $i^{\text {th }}$ row and $j^{\text {th }}$ column of $I_{p}$, obtaining

$$
M_{i j}^{3}(\Lambda)=\left[\begin{array}{ccccccccc}
I_{1} & \cdots & 0 & 0 & \cdots & 0 & 0 & \cdots & 0 \\
0 & \ddots & 0 & 0 & \cdots & 0 & 0 & \cdots & 0 \\
0 & \cdots & I_{i} & 0 & \cdots & 0 & \Lambda & \cdots & 0 \\
0 & \cdots & 0 & \ddots & \cdots & 0 & 0 & \cdots & 0 \\
0 & \cdots & 0 & 0 & I & 0 & 0 & \cdots & 0 \\
0 & \cdots & 0 & 0 & \cdots & \ddots & 0 & \cdots & 0 \\
0 & \cdots & 0 & 0 & \cdots & 0 & I_{j} & \cdots & 0 \\
0 & \cdots & 0 & 0 & \cdots & 0 & 0 & \ddots & 0 \\
0 & \cdots & 0 & 0 & \cdots & 0 & 0 & \cdots & I_{m}
\end{array}\right] .
$$

\subsubsection{The GEROME-B Algorithm}

In the scalar case, the Gauss-Jordan elimination acts using pivots to eliminate the other elements on the same column. Similarly, our extension needs block pivot elements. They must follow the next

Definition 1. A block of the matrix $P$ can be a pivot if and only if

- it is a full-rank square block,

- it is the only pivot in its row and column,

- it does not multiply an independent variable of the system.

The block elimination procedure, called GEROME-B: Gauss Elementary Row Opperation Method for Block Partitioned Matrix, is explained in Algorithm 1. Without losing generality, we suppose to act on a particular permutation $\hat{P}$ of the initial matrix $P$, obtained using matrices of the type (40), where $\hat{P}$ has all the pivots on the main diagonal, and the blocks multiplying the input variables in the last columns. When the algorithm is completed, the permutation can be inverted to replace pivots and variables in the starting order.

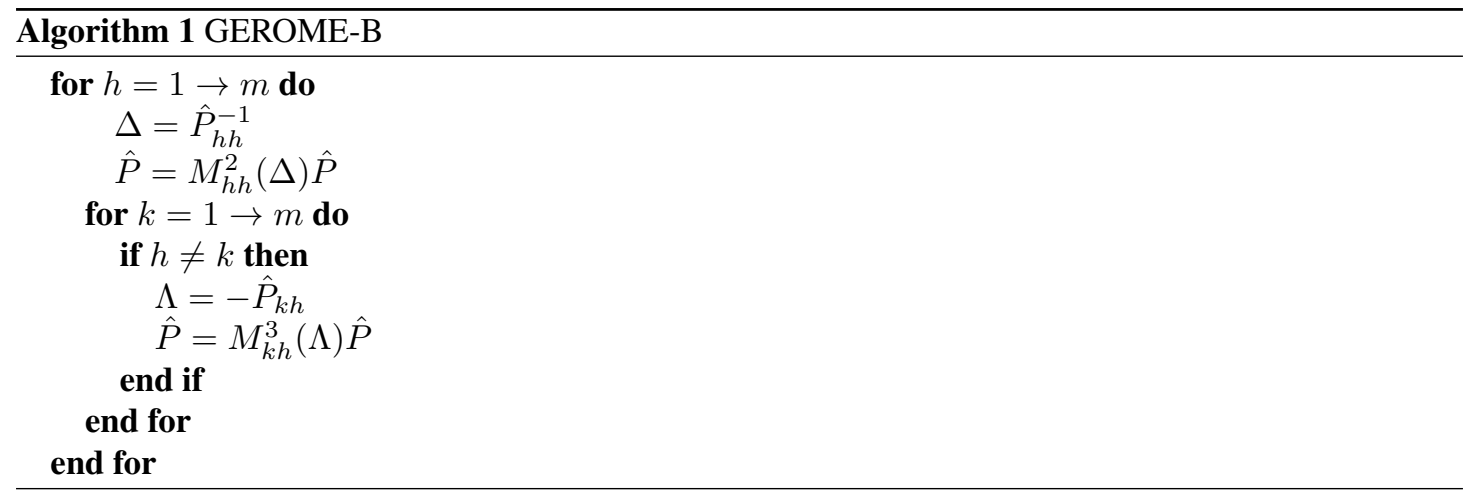

In order to apply Algorithm 1 to the Fundamental Grasp Matrix in (33), permutations are not needed if we take the elements on the main diagonal as pivots. In fact, in this case all the blocks on the main diagonal are square and full rank matrices. The only exception is the fourth row, where a null pivot element appears. Unfortunately, this is an obliged choice, because that is the only square matrix in its own column. To tackle this problem, we can consider the following steps: 
1. pre-multiplying the Fundamental Grasp Matrix for $M_{41}^{3}\left(-{ }^{b} G\right)$, this is equivalent to substitute the fourth row with the fourth row minus ${ }^{b} G$ times the first row,

2. applying the matrix $M_{45}^{3}\left(-{ }^{b} G K_{c}\right)$, this is equivalent to substitute the (new) fourth row with the fourth row minus ${ }^{b} G K_{c}$ times the fifth row,

3. applying the matrix $M_{46}^{3}\left({ }^{b} G K_{c}\right)$, this is equivalent to substitute the (new) fourth row with the fourth row plus ${ }^{b} G K_{c}$ times the sixth row.

Applying these operations, from (33) we obtain the form

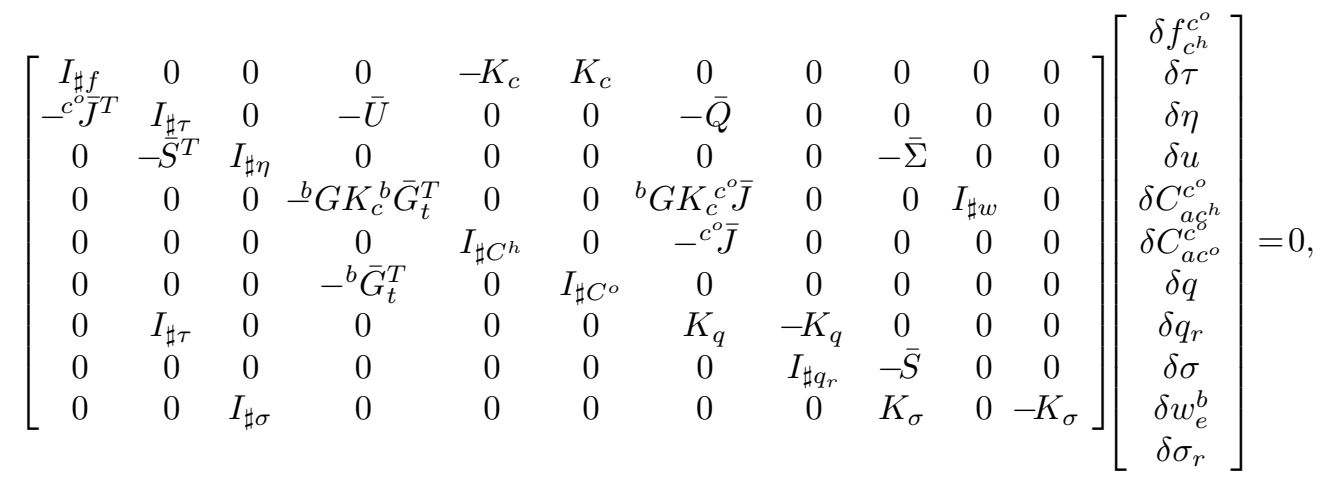

briefly indicated as $A_{1} \delta y=0$.

\subsection{The Fundamental Grasp Matrix in Canonical Form}

The new coefficient matrix $A_{1}$ in (43) satisfies all the necessary hypothesis to apply Algorithm 1, in terms of pivots collocation and rank. Before seeing the final result, we want to underline the following

Remark 1. GEROME-B allows us to obtain a new suitable form of the coefficient matrix. This corresponds to a new set of equations, where each dependent variable is a function only of the independent ones. If the pivots are on the main diagonal, the result is a quasi-diagonal matrix, or, otherwise, a general permutation of a quasi-diagonal matrix.

From previous considerations, it is clear that applying the Algorithm 1 to the coefficient matrix in (43), we obtain the form

$$
\left[\begin{array}{ccccccccccc}
I_{\sharp f} & 0 & 0 & 0 & 0 & 0 & 0 & 0 & 0 & W_{f} & R_{f} \\
0 & I_{\sharp \tau} & 0 & 0 & 0 & 0 & 0 & 0 & 0 & W_{\tau} & R_{\tau} \\
0 & 0 & I_{\sharp \eta} & 0 & 0 & 0 & 0 & 0 & 0 & W_{\eta} & R_{\eta} \\
0 & 0 & 0 & I_{\sharp u} & 0 & 0 & 0 & 0 & 0 & W_{u} & R_{u} \\
0 & 0 & 0 & 0 & I_{\sharp C^{h}} & 0 & 0 & 0 & 0 & W_{C^{h}} & R_{C^{h}} \\
0 & 0 & 0 & 0 & 0 & I_{\sharp C^{o}} & 0 & 0 & 0 & W_{C^{o}} & R_{C^{o}} \\
0 & 0 & 0 & 0 & 0 & 0 & I_{\sharp q} & 0 & 0 & W_{q} & R_{q} \\
0 & 0 & 0 & 0 & 0 & 0 & 0 & I_{\sharp q_{r}} & 0 & W_{q_{r}} & R_{q_{r}} \\
0 & 0 & 0 & 0 & 0 & 0 & 0 & 0 & I_{\sharp \sigma} & W_{\sigma} & R_{\sigma}
\end{array}\right]\left[\begin{array}{c}
\delta f_{c^{h}}^{c^{o}} \\
\delta \tau \\
\delta \eta \\
\delta u \\
\delta C_{a c^{c}}^{c^{h}} \\
\delta C_{a c^{c}}^{c^{c}} \\
\delta q \\
\delta q_{r} \\
\delta \sigma \\
\delta w_{e}^{b} \\
\delta \sigma_{r}
\end{array}\right]=0 .
$$

Equation (44) is briefly expressed as $A_{c} \delta y=0$, where $A_{c} \in \mathbb{R}^{r_{a} \times c_{a}}$, is the Canonical Form of the Fundamental Grasp Matrix (cFGM). For the reader convenience, we refer to the Appendix D for the complete expression of the matrices $W_{\nu}$ and $R_{\nu}$. 
It is worth observing that the previous results are relevant especially considering an application in symbolic form. In fact, similar conclusions are not difficult to achieve with a numerical approach. In this sense, using the subscript $d$ indicating portions relative to the dependent variables, and $i$ to the independent ones, we can observe that (33) can be expressed even as

$$
A \delta y=\left[\begin{array}{ll}
A_{d} & A_{i}
\end{array}\right]\left[\begin{array}{l}
\delta y_{d} \\
\delta y_{i}
\end{array}\right]=0
$$

where $A_{d} \in \mathbb{R}^{r_{a} \times r_{a}}, A_{i} \in \mathbb{R}^{r_{a} \times\left(c_{a}-r_{a}\right)}, \delta y_{d} \in \mathbb{R}^{r_{a}}, \delta y_{i} \in \mathbb{R}^{\left(c_{a}-r_{a}\right)}$. Under the same previous hypothesis, the canonical form of the system can be numerically obtained premultiplying (45) for $A_{d}^{-1}$, as follows

$$
A_{d}^{-1}\left[\begin{array}{ll}
A_{d} & A_{i}
\end{array}\right]\left[\begin{array}{l}
\delta y_{d} \\
\delta y_{i}
\end{array}\right]=\left[\begin{array}{ll}
I & A_{d}^{-1} A_{i}
\end{array}\right]\left[\begin{array}{c}
\delta y_{d} \\
\delta y_{i}
\end{array}\right]=A_{c} \delta y=0
$$

\section{RELEVANT PROPERTIES OF THE FUNDAMENTAL GRASP MATRIX IN CANONICAL FORM}

In the next sections, we will analyse the relevant properties of some blocks in (44). For the sake of completeness, in Appendix D the cases of a grasp performed by (i) a fully actuated hand, (ii) a hand equipped with elastic joints and (iii) a soft synergy underactuated hand are considered. The expression of the elements of the canonical forms of the Fundamental Grasp Matrices are presented there for all the cases.

\subsection{Controllable Internal Forces}

From (44), we consider the expression of the contact force variation, that is

$$
\delta f_{c^{h}}^{c^{o}}+W_{f} \delta w_{e}^{b}+R_{f} \delta \sigma_{r}=0
$$

Defining as internal the solutions of (44), or equivalently of (33), not involving the external wrench variation, it immediately follows that the span of the matrix $R_{f}$ is the subspace of controllable internal forces.

\subsection{Contact Force Transmission Caused by an External Wrench}

Again from (47), we note that $W_{f}$ is a map from the external wrench to the contact forces, when the inputs on the hand are kept constant. Therefore, $-W_{f}$ represents the contact force transmission of the external wrench.

Both controllable internal forces and contact force distribution have great relevance in problems as the contact force optimization and the evaluation of the force closure properties of the grasp.

\subsection{Controllable Internal Object Displacements}

Especially in the case of underactuated hands, it could be tricky to find what kind of motion the hand can impose to the object. This problem is solved by considering the fourth equation of (44), that give us a description of the object displacements as

$$
\delta u+W_{u} \delta w_{e}^{b}+R_{u} \delta \sigma_{r}=0 .
$$

Easily we can deduce that the range space of the matrix $R_{u}$ represent the subspace of the controllable internal object displacements. 


\subsection{Grasp Compliance}

Again from (48), we can find that $-W_{u}$ is the grasp compliance. In other words, $C_{g}=-W_{u}$ represents the compliance that a $6 D$ spring should have to behave like the hand, maintaining the same object displacement consequent to an external wrench variation.

It is worth observing on passing that, applying Algorithm 1 to (33), substituting the displacement $\delta u$ to the wrench $\delta w_{e}^{b}$ as input variable, we would have dually found the grasp stiffness.

\section{MAIN CATEGORIES OF MANIPULATION TASKS}

In the previous sections, we have shown how to describe the system and to determine a perturbed configuration consequent to an input variable variation. Algorithm 1 conducted us to find some particular subsets of solutions of the system, e.g. the controllable internal forces. This suggested the idea to search other possible relevant solution subsets. In this way, we will show how to define some relevant types of grasps, based on the nullity or non-nullity patterns of the system variables. However, the given definitions involve only some variables. In Section 7, we will proceed to discover all the nullity patterns that could be present inside the solution set of (33).

To help us in a clear exposition of reasoning and results, we introduce a specific notation. For a variable variation $\delta \nu \in \mathbb{R}^{\sharp \nu}$ we could have the cases:

- $\delta \nu=0$, if all the elements of the vector are null,

- $\delta \nu=*$, if at least one element of the vector is non-null,

- $\delta \nu=\circledast$, if the vector can be indifferently null or not.

\subsection{Internal Forces and Displacements}

As explained in Section 4, a variation of the augmented configuration is called internal ${ }^{2}$ if it does not involve any variation of the external wrench acting on the object, that is

$$
\delta w_{e}^{b}=0
$$

\subsection{External Forces and Displacements}

On the contrary, a perturbation $\delta y$ is called external if it involves a variation of the external wrench acting on the object, that is

$$
\delta w_{e}^{b}=*
$$

\subsection{Pure Squeeze}

We define the pure squeeze case as the situation in which the hand perform a contact force variation on the object, without moving it. Synthetically, the pure squeeze occurs when

$$
\left\{\begin{array}{l}
\delta w_{e}^{b}=0 \\
\delta f_{c^{h}}^{c^{o}}=* \\
\delta u=0 .
\end{array}\right.
$$

\footnotetext{
${ }^{2}$ It is worth remarking that our definition of internal forces implies the use of object frame components for the external wrench. This choice allows to filter out the influence of object motions that, otherwise, would have come across in a spatial description of the same quantity.
} 


\subsection{Spurious Squeeze}

If the contact force variation is accompanied by an object displacements, we will talk of spurious squeeze. This case corresponds to have

$$
\left\{\begin{array}{l}
\delta w_{e}^{b}=0 \\
\delta f_{c^{c}}^{c^{o}}=* \\
\delta u=* .
\end{array}\right.
$$

\subsection{Kinematic Grasp Displacements}

Still inside the subspace of internal forces and displacements, we identify those perturbations $\delta y$ that do not involve any violation of the (rigid) kinematic contact constraints. Equivalently, those perturbations that do not involve any variation of the elastic potential energy stored in the contact springs, with respect to the reference configuration. This effect is obtained with solutions involving object displacements, but no contact force variation. Synthetically, we look for solutions in the form

$$
\left\{\begin{array}{l}
\delta w_{e}^{b}=0 \\
\delta f_{c^{h}}^{c^{o}}=0 \\
\delta u=* .
\end{array}\right.
$$

It is worth observing that, if we regard the displacements of the extremities of the contact springs as descriptive of elastic deformation of the grasped object and of the fingertip, the kinematic grasp, requiring a null variation of contact forces, implies a null variation of the object shape after the displacement. In this interpretation, the definition of rigid object displacements can be recovered. We want to remark that, in order to give consistent definitions, it is necessary to describe the contact forces in the object reference frame. Otherwise, describing forces in the absolute frame, not all the contact force variations necessarily corresponds to contact spring (or object shape) deformations. A simple example of this fact is the pure rotation of the grasped object. To preserve its shape, the initial contact forces have to rotate with it. Thus, if contact forces were expressed in an absolute frame, a variation would have occurred just because of the relative rotation.

\subsection{Redundant Motion of the Hand}

It could be in interest to find the motions of the hand not involving the object, e.g. to move the joints away from the limit values. This corresponds to the conditions

$$
\left\{\begin{array}{l}
\delta w_{e}^{b}=0 \\
\delta f_{c^{o}}^{c^{o}}=0 \\
\delta u=0 \\
\delta q=* .
\end{array}\right.
$$

For the hand, this kind of movements represent to the so-called nullspace motions. Nevertheless, in the rest of the paper, we will not use this name in order to avoid confusion with other definitions.

\subsection{External Structural Forces}

In the subspace of external actions, it is possible to look for perturbations $\delta y$ that are characterized by variations of contact forces $\delta f_{c h}^{c^{\circ}}$, but do not involve any modifications of the synergistic actions and references. This can be equivalently described as

$$
\left\{\begin{array}{l}
\delta w_{e}^{b}=* \\
\delta f_{c^{o}}^{c^{o}}=* \\
\delta \eta=0 \\
\delta \sigma_{r}=0 .
\end{array}\right.
$$




\subsection{Resultant Decomposition of the Solution Space}

The previous definitions correspond to particular forms of solutions for the system (33). Since the basis $\Gamma$ in (36) contains all the solution of the system, even this particular forms can be searched there. This corresponds to obtaining a decomposition of the solution space as follows

$$
\left[\begin{array}{c}
\delta f_{c^{h}}^{c^{\prime}} \\
\delta \tau \\
\delta \eta \\
\delta u \\
\delta C_{a c^{c^{h}}} \\
\delta C_{a c^{o}}^{c^{\circ}} \\
\delta q \\
\delta q_{r} \\
\delta \sigma \\
\delta w_{e}^{b} \\
\delta \sigma_{r}
\end{array}\right]=\left[\begin{array}{ccccccc}
\circledast & * & * & 0 & 0 & * & \circledast \\
\circledast & \circledast & \circledast & \circledast & \circledast & \circledast & \circledast \\
\circledast & \circledast & \circledast & \circledast & \circledast & 0 & \circledast \\
\circledast & 0 & * & * & 0 & \circledast & \circledast \\
\circledast & \circledast & \circledast & \circledast & \circledast & \circledast & \circledast \\
\circledast & \circledast & \circledast & \circledast & \circledast & \circledast & \circledast \\
\circledast & \circledast & \circledast & \circledast & * & \circledast & \circledast \\
\circledast & \circledast & \circledast & \circledast & \circledast & \circledast & \circledast \\
\circledast & \circledast & \circledast & \circledast & \circledast & \circledast & \circledast \\
0 & 0 & 0 & 0 & 0 & * & * \\
\circledast & \circledast & \circledast & \circledast & \circledast & 0 & \circledast
\end{array}\right] x .
$$

As we can see, each column (from the $2^{\text {nd }}$ one to the $6^{\text {th }}$ one) corresponds to a definition given above. Exceptions are the first and the last column, that are introduced to complete the space of internal and external forces respectively.

The careful reader will observe that from some nullity patterns previous defined, taking into account (33), it can follow that other variables are necessarily null or non-null. This fact will be analysed in Section 7, where all the constraints expressed by (33) will be taken into account to define a complete and consistent taxonomy (classification) of the solution space. Here we want to focus the attention only on the necessary conditions on which the definitions hold.

\section{BLOCK DECOMPOSITION OF THE NULLSPACE MATRIX}

From linear algebra, it is known that every matrix $C \in \mathbb{R}^{r_{c} \times c_{c}}$, with $\rho_{c}=\operatorname{rank}(C)$, can be expressed, by a Gauss-Jordan elimination, in its reduced row echelon form (RREF), via a suitable permutation matrix $\Pi \in \mathbb{R}^{r_{c} \times r_{c}}$, such that

$$
\Pi C=\left[\frac{U}{0}\right],
$$

where $U \in \mathbb{R}^{\rho_{c} \times c_{c}}$ is a staircase matrix, and the zero block has consequent dimensions. It is worth recalling that the reduced row echelon form of a given matrix is unique. The reader can find other details about the RREF in [Meyer, 2000].

As explained in [Bicchi et al., 1995], this particular form can be used to search nullity patterns, as in (56), inside general matrices. In order to explain the method, we show operatively the details of each step to find a basis for internal solution of the system.

In later discussion, we will use the assumption to have access to a function $\operatorname{rref}(X)$ able to return the reduced row echelon form of its argument $X^{3}$. The procedure to define a basis for the internal system perturbations $\left(\delta w_{e}^{b}=0\right)$, consists of the steps: (i) partitioning the basis of the solution space $\Gamma$, isolating the portion relative to the variable that we want to nullify (or not nullify). In the example of internal actions, from (37), we obtain

$$
\delta y=\left[\begin{array}{c}
\Gamma_{\tilde{w}_{1}} \\
\Gamma_{w} \\
\Gamma_{\tilde{w}_{2}}
\end{array}\right] x,
$$

${ }^{3}$ This is a typical situation using the most popular computational platforms, e.g.: $\operatorname{rre} f(X)$ in MATLAB and RowReduce (X) in Mathematica. 
where the symbols $\Gamma_{\tilde{w}_{1}}$ and $\Gamma_{\tilde{w}_{2}}$ correspond to blocks that are not relative to the external wrench variable. From this, we extract the block $\Gamma_{w} \in \mathbb{R}^{\sharp w \times c_{\gamma}}$ relative to the external wrench; (ii) obtaining a permutation matrix. As a consequence of the properties of the RREF, detailed in [Meyer, 2000], running the function $\operatorname{rref}\left(\left[\Gamma_{w}^{T} \mid I_{c_{\gamma}}\right]\right)$, where $I_{c_{\gamma}} \in \mathbb{R}^{c_{\gamma} \times c_{\gamma}}$ is an identity matrix, the result is $\left[\frac{U_{w}}{0} \mid \Pi_{w}\right]$, where $U_{w} \in \mathbb{R}^{\rho_{w} \times \sharp w}$, with $\rho_{w}=\operatorname{rank}\left(\Gamma_{w}\right)$. The block $\Pi_{w} \in \mathbb{R}^{c_{\gamma} \times c_{\gamma}}$ is the permutation matrix such that $\Pi_{w} \Gamma_{w}^{T}=U_{w}$; (iii) using these results to find the new form ${ }^{1} \Gamma \in \mathbb{R}^{r_{\gamma} \times c_{\gamma}}$ for the basis $\Gamma$ as

$$
{ }^{1} \Gamma=\Gamma \Pi_{w}^{T}=\left[\begin{array}{c}
{ }^{1} \Gamma_{\tilde{w}_{1}} \\
\hline{ }^{1} \Gamma_{w} \\
\hline{ }^{1} \Gamma_{\tilde{w}_{2}}
\end{array}\right],
$$

where, in detail, ${ }^{1} \Gamma_{w}=U_{w}^{T},{ }^{1} \Gamma_{\tilde{w}_{1}}=\Gamma_{\tilde{w}_{1}} \Pi_{w}^{T}$, and ${ }^{1} \Gamma_{\tilde{w}_{2}}=\Gamma_{\tilde{w}_{2}} \Pi_{w}^{T}$.

By the way, we observe that the matrix ${ }^{1} \Gamma$ is essentially a permutation and combination of the columns of $\Gamma$. This assures that the two matrices have the same column space, or, in other words, that both are a solution basis for the system (33).

With simple considerations, it is evident that (59) can be written as

$$
{ }^{1} \Gamma=\left[{ }^{1} \Gamma_{\text {ext }}{ }^{1} \Gamma_{\text {int }}\right],
$$

where ${ }^{1} \Gamma_{\text {ext }} \in \mathbb{R}^{r_{\gamma} \times \rho_{w}}$ and ${ }^{1} \Gamma_{\text {int }} \in \mathbb{R}^{r_{\gamma} \times\left(c_{\gamma}-\rho_{w}\right)}$, that result a basis for external and internal solution respectively. Thus we have obtained the desired result.

Similarly, the procedure can be iterated to discover other subspaces defined in previous sections, searching nullity or not-nullity of the variable of interest each time.

\section{COMPLETE TAXONOMY OF THE GRASP}

In Section 5 some relevant kinds of system configuration variations are defined by imposing to some variables to be null or non-null. This approach can be further investigated, arriving to discern all the combinations of null or non-null variables, which can compose a feasible solution of the system (33).

To this aim, we will use a sort of sieve of Eratosthenes [Cojocaru et al., 2005]: starting from all the possible combinations, we will proceed eliminating those forbidden by the equations composing (33).

To easily identify the forbidden solutions, it is useful to reorganize the system variables in levels as follows:

- object level, involving the external wrench $\delta w_{e}^{b}$, and the object displacement $\delta u$,

- hand/object interface level, involving the contact forces $\delta f_{c^{h}}^{c^{\circ}}$, the displacement of the object contact frame $\delta C_{a c^{o}}^{c^{o}}$, and the displacement of the hand contact frame $\delta C_{a c^{h}}^{c^{o}}$,

- hand level, involving the joint torques $\delta \tau$, the joint configuration $\delta q$, and the joint reference configuration $\delta q_{r}$,

- synergy level, involving the synergy forces $\delta \eta$, the synergy configuration $\delta \sigma$, and synergy references $\delta \sigma_{r}$.

\subsection{In-Level Constraints}

Since a grasp performed by a synergistic underactuated hand is described by eleven variables, restricting the discussion to consider the possibility that they can only be null or non-null, all the possible combinations are $2^{11}=2048$. However, each equation composing (33) can be interpreted as a constraint that the feasible nullity patterns have to satisfy. In other words, as we will show shortly, some combinations of null or non-null variables are forbidden by the equations of the system. These combinations can be found and eliminated in order to bring out all the combinations allowed. 


\begin{tabular}{c|cc}
\hline & $\delta w_{e}^{b}$ & $\delta u$ \\
\hline$O . A$ & $*$ & $*$ \\
$O . B$ & $*$ & 0 \\
$O . C$ & 0 & $*$ \\
$O . D$ & 0 & 0 \\
\hline
\end{tabular}

Table 2: Object level combinations.

In Table 2 we present all the possible (non-)nullity combinations involving the object level variables. By comparison with equations grouped in (33), it results that no combination is forbidden.

In Table 3, we represent all the possible combinations for the variables at the hand/object interface level. Taking into account (25), from an algebraic point of view, under the assumption that the contact stiffness matrix $K_{c}$ is square and full rank, it results that it is not possible to have a contact force without any contact frame displacements. Similarly, it is not possible to have a displacement of only one contact frame, without contact forces.

In other terms, from a physical point of view, the combination $I . D$ corresponds to a contact force variation that appears without a corresponding deformation of the virtual contact spring. On the contrary, the combinations I.F and I.G describe situations where the contact spring is necessarily deformed, since one contact frame is fixed but the other moves; despite this fact no contact forces are there. Therefore it is clear that the combinations I.D, I.F, and I.G, corresponding to situations without physical meaning, are not allowed (highlighted in grey in Table 3).

\begin{tabular}{c|ccc}
\hline & $\delta f_{c^{h}}^{c^{\circ}}$ & $\delta C_{a c^{\circ}}^{c^{\circ}}$ & $\delta C_{a c^{h}}^{c^{\circ}}$ \\
\hline$I . A$ & $*$ & $*$ & $*$ \\
$I . B$ & $*$ & $*$ & 0 \\
$I . C$ & $*$ & 0 & $*$ \\
$I . D$ & $*$ & 0 & 0 \\
$I . E$ & 0 & $*$ & $*$ \\
$I . F$ & 0 & $*$ & 0 \\
$I . G$ & 0 & 0 & $*$ \\
$I . H$ & 0 & 0 & 0 \\
\hline
\end{tabular}

Table 3: Hand/object interface level combinations.

Even for the hand level, whose (non-)nullity combinations are in Table 4, similar considerations hold. Equation (27) enables us to eliminate the cases H.D, H.F and H.G, if the joint stiffness matrix is square and full rank. As similarly discussed for the hand/object interface level, the combinations forbidden by (27) correspond to the presence of a joint torque variation without changing neither joint nor joint reference configuration (H.D), and, on the contrary, a variation of the joint actuation spring length, without a corresponding torque variation (H.F and H.G). With a similar physical motivation, we can eliminate the cases $S . D, S . F$ and $S . G$ from Table 5, under the assumption that the synergy stiffness matrix in (32) is square and full rank.

\subsection{Intra-Level Constraints}

With the previous discussion, we have considerably reduced the number of acceptable combinations. In fact, only four possibilities for the object level and five for all the other three levels remain. The total amount is now $4 \times 5^{3}=500$ possible combinations. But also the other equations of the system (33) can be taken into account, bringing us to eliminate some interconnection between the levels. In fact, from (8), we can observe that, if the grasp matrix ${ }^{b} G$ is full row-rank, as it is usually the case, it is not possible to have 


\begin{tabular}{c|ccc}
\hline & $\delta \tau$ & $\delta q$ & $\delta q_{r}$ \\
\hline$H . A$ & $*$ & $*$ & $*$ \\
$H . B$ & $*$ & $*$ & 0 \\
$H . C$ & $*$ & 0 & $*$ \\
$H . D$ & $*$ & 0 & 0 \\
$H . E$ & 0 & $*$ & $*$ \\
$H . F$ & 0 & $*$ & 0 \\
$H . G$ & 0 & 0 & $*$ \\
$H . H$ & 0 & 0 & 0 \\
\hline
\end{tabular}

Table 4: Hand level combinations.

\begin{tabular}{c|ccc}
\hline & $\delta \eta$ & $\delta \sigma$ & $\delta \sigma_{r}$ \\
\hline$S . A$ & $*$ & $*$ & $*$ \\
$S . B$ & $*$ & $*$ & 0 \\
$S . C$ & $*$ & 0 & $*$ \\
$S . D$ & $*$ & 0 & 0 \\
$S . E$ & 0 & $*$ & $*$ \\
$S . F$ & 0 & $*$ & 0 \\
$S . G$ & 0 & 0 & $*$ \\
$S . H$ & 0 & 0 & 0
\end{tabular}

Table 5: Synergy level combinations.

an external wrench variation and a null variation of the contact force. This means that the groups O.A and $O . B$ can be present in combination with the groups $I . A, I . B, I . C$ and $I . D$ (the last one eliminated already in Table 3), but not with the others. The cases eliminated correspond to situations in which an external wrench would not have an effect on the contact. In other words, we restrict our analysis just to the cases of isostatic and hyperstatic equilibrium of the object, thus to situation in which the number of independent constraints imposed on the object by the interaction with the hand is equal or greater than its degrees of freedom. Similarly, from (14) it follows that, in the presence of an object contact frame displacement $\delta C_{a c^{o}}^{c^{o}}$, there is always an object displacements $\delta u$. This corresponds to assuming that every object displacement has effect on at least one virtual contact spring.

From these considerations, it results that

- the group O.A can occur only in conjunction with $I . A$ or $I . B$,

- the group O.B can occur only in conjunction with $I . C$,

- the group O.C can occur only in conjunction with I.A, I.B or I.E,

- the group O.D can occur only in conjunction with I.C or I.H.

The variables of the interface and the hand level are related by (19). To satisfy this relationship, it is necessary that, if the variable $\delta C_{a c^{h}}^{c^{o}}$ is non-zero, also $\delta q$ be non-zero. This is equivalent to impose that every hand contact frame displacements is caused by a hand configuration variation. Instead, the possibility to have a joint movement not involving a hand contact frame displacement is allowed. This situation corresponds to reconfiguration motions of the hand. Then it follows that

- the group I.A can occur only in conjunction with H.A,H.B or H.E,

- the group I.B can occur in conjunction with H.A, H.B, H.C, H.E or H.H (all the hand level combinations allowed), 
- the group $I . C$ can occur only in conjunction with H.A,H.B or H.E,

- the group $I . E$ can occur only in conjunction with H.A,H.B or H.E,

- the group $I . H$ can occur in conjunction with H.A,H.B,H.C, H.E or $H . H$ (all the hand level combinations allowed),

Equations (29) and (31) relate the hand level with the synergy level variables. Assuming that the synergy matrix is full column rank, we eliminate all the combinations in which a joint reference displacements is present without a synergistic variation, and vice versa. The connections admitted can be summarized as

- the group $H . A$ can occur only in conjunction with $S . A, S . B$ or $S . E$,

- the group $H . B$ can occur only in conjunction with $S . C$ or $S . H$,

- the group H.C can occur only in conjunction with S.A, S.B or S.E,

- the group $H . E$ can occur only in conjunction with $S . A, S . B$ or $S . E$,

- the group $H . H$ can occur only in conjunction with S.H.

Finally, equation (23) relates three levels and impose that, if $\delta \tau$ is non-null, the variables $\delta u, \delta f_{c^{h}}^{c^{o}}$ and $\delta q$ cannot be null at the same time or, in other words, impose that the presence of a joint torque variation needs an explanation in a contact force or in a change of configuration. Taking into account the previously found constraints, it follows that it is not possible to get the condition $O . D$ in conjunction with $I . H$ and $H . C$ at the same time.

The total amount of the remaining combinations is 57 , or 56 without considering the trivial solution composed by the combination $O . D, I . H, H . H$ and $S . H$, where no displacement of the system actually exists.

\subsection{The Taxonomic Labyrinth}

The set of all the acceptable combinations of the groups presented in Tables 2-5 define the complete taxonomy of the grasp with soft synergy underactuation, sketched in Figure 2. Despite the fact that the figure appears as a labyrinth, it is not difficult to read it. It is possible to start indifferently from a group located at the top or at the bottom level. From this, one can move toward the next level, downward or upward respectively, moving from the bubble containing the starting group (e.g. $O . B$ or S.E). Each of these bubbles has a square door that allows to access another bubble, in the next level, containing all the admissible groups that can be chosen. From the new group, all the bubbles containing it define a new set of possible doors one can use, considering that it is forbidden to go back to the previous level.

From the chosen door, we can access to a new bubble, where a new group can be chosen. The process is then repeated, until arriving to the last level, with the sole limitation that it is not possible to have together all the elements O.D, I.H and H.C (in a dotted box in Figure 2).

As a simple example, let us explain how to search the redundant motion of the hand, defined in (54). From the conditions on the external wrench $\left(\delta w_{e}^{b}=0\right)$ and on the object displacement $(\delta u=0)$, by a direct comparison with Table 2, it follows that we can start from the element $O . D$, on the top level of the Figure 2. The square door, connecting to the hand/object interface level, allows the access to the groups $I . C$ and $I . H$. By their definitions, in Table 3 , we note that the group $I . C$ requires a contact force variation $\left(\delta f_{c^{h}}^{c^{o}}=*\right)$, while for the group $I . H$ holds that $\delta f_{c^{h}}^{c^{o}}=0$. By the definition in (54), the condition on the interface level $\left(\delta f_{c^{h}}^{c^{o}}=0\right)$ is satisfied only choosing the group I.H. Considering the bubble containing it, the door allows to choose every element of the hand level. Again from (54), for the hand level variables we have to guarantee that a non-null joint displacement $(\delta q=*)$, thus only the groups $H . A, H . B$ or H.E are acceptable. To make things clearer, we take the group $H . E$, corresponding to a particular redundant motion of the hand that does not require to change the joint torques. If no other condition is needed, 


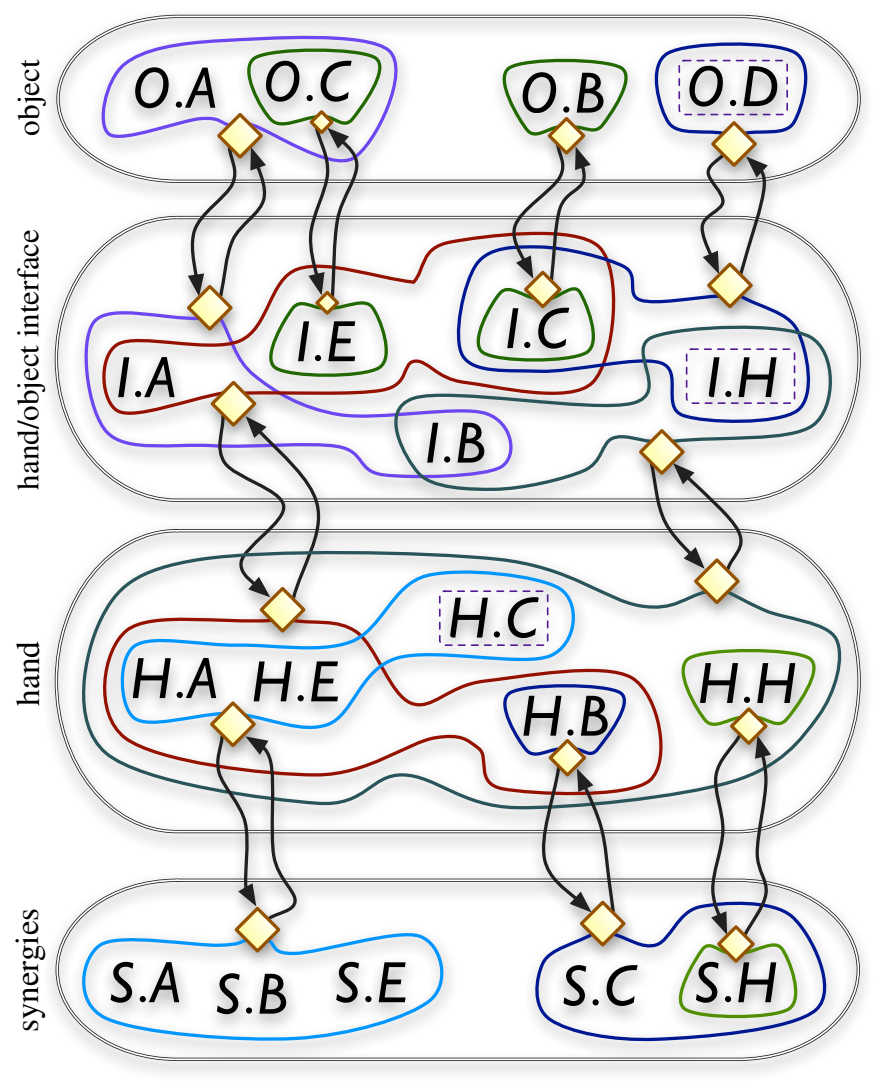

Figure 2: Complete taxonomy of the grasp.

finally we find that we can connect to $S . A, S . B$ or $S . E$. By a comparison with Table 5 , we can give an interpretation of these three cases. While the group $S . A$ correspond to the most general case, where all synergistic actuation is involved, the other two cases deserve a more detailed discussion. A solution of the system including the group $S . B$ indicates the presence of an equilibrium configuration of the system where the synergy reference configuration is held changing the generalized forces at the synergy level. The situation can occur in the case the force transmission ratio between the joint torques and synergistic forces can vary with the synergy configuration. The presence of a solution including the group $S . E$, indeed, points out the possibility to reconfigure the synergy actuation, without changing the generalized forces provided at the synergy level.

\section{OTHER TYPE OF (UNDER-)ACTUATION}

The soft synergy underactuation mechanism is currently attracting much attention and interest. However, we cannot ignore that it is not the only actuation mechanism worth studying. Over the obvious interest to study fully actuated robotic hands, other actuation approaches deserve attention and need suitable analysis tools, as for instance eigengrasps [Ciocarlie et al., 2007], parallel structure based [Birglen et al., 2008] and adaptive synergies [Catalano et al., 2012]. Despite the fact that some parts of this paper concern strictly the soft synergy underactuation, the framework presented is general enough to cope with the above mentioned underactuated situations.

The basic requirement to apply the methods we propose, is to write all the equations describing the kineto-static behavior of the particular system under investigation. Casting together all the relationships, 
we basically obtain a new type of Fundamental Grasp Matrix, for which it is straightforward how to apply GEROME-B and Find-X methods. Similarly, the decomposition of the nullspace by using the RREF, can be performed. However, it is necessary to pay attention when classifying the main categories of manipulation tasks. The definitions proposed, basically involve the variables from the object to the hand level, and these can be used for any kind of (under-)actuation considered. One exception is the subspace of the external structural forces. We can simply recover this concept considering instead the conditions

$$
\left\{\begin{array}{l}
\delta w_{e}^{b}=* \\
\delta f_{c^{h}}^{c^{o}}=* \\
\delta \tau^{\star}=0 \\
\delta q^{\star}=0,
\end{array}\right.
$$

where $\delta q^{\star}$ and $\delta \tau^{\star}$ are the generalized displacements and forces at the (under-)actuation level for the present case.

As a simple example, the FGM for the case in which no underactuation is considered on the hand can be obtained taking (8), (14), (19), (23), (25), as in (121). The presence of elastic actuators can be introduced in the previous model, restoring (27), as in (125). For these examples, the block elements composing the canonical form of the FGM are described in the Appendix D. As a final example, the eigengrasp case can be considered adding, to the system describing the fully actuated case (121), expressions of the type (29) and (31), but involving the joint displacements $\delta q$ instead of the references $\delta q_{r}$, that is in the form

$$
\begin{gathered}
\delta q=S(\sigma) \delta \sigma \\
\delta \eta=S^{T}(\sigma) \delta \tau+\Sigma(\sigma, \tau) \delta \sigma .
\end{gathered}
$$

After the proper FGM for the system in interest is built, taking into account the previous considerations, the approaches proposed in this paper can be adopted to obtain symbolic relationships from the input and the output variables (GEROME-B). Computationally efficient methods to find whole system perturbations (Find-X), and main categories of the manipulation tasks (nullspace block decomposition by the use of the RREF) can again be applied.

\section{NUMERICAL EXAMPLES}

\subsection{Precision Grasp}

Let us consider the 2D example shown in Figure 3, where a two fingered hand is grasping a circular object of radius $R$ with its fingertips. Each finger is composed by two links of length $L$. Globally, the hand has four revolute joints $\left[j_{1}, \ldots, j_{4}\right]$. Attached to the palm we fix an inertial frame $\{A\}$ with origin on the intersection between the axis of $j_{1}$ and the plane of the figure. On the $k^{\text {th }}$ contact point we introduce two reference frames $\left\{C_{k}^{h}\right\}$ and $\left\{C_{k}^{o}\right\}$ fixed with the hand and the object, respectively, aligned with $\{A\}$ in the reference configuration. Attached to the object we pose a frame $\{B\}$ with origin in the center of the circle, initially parallel to $\{A\}$. The generic displacement of $\{B\}$ with respect to $\{A\}$ is described by the vector $\delta u^{T}=\left[\delta u_{x}^{T}, \delta u_{y}^{T}, \delta u_{\alpha}^{T}\right]^{T}$, where the first two elements indicate a linear displacement along the axes $x$ and $y$, respectively, and the last one an angular displacement around the axis $z$. It is worth observing that the displacement of the object is described in coordinates expressed in $\{A\}$. As numerical values for the parameters of the problem we consider $L=R=1 \mathrm{~m}, k_{q}=10 \mathrm{~N} / \mathrm{rad}, k_{c}=10 \mathrm{~N} / \mathrm{m}$, $k_{s}=10 \mathrm{~N} / \mathrm{m}$, and we assume the preload $\bar{f}_{c^{o}}^{c^{h}}=[1,1,-1,1]^{T} \mathrm{~N}$, as sketched in Figure 3 . By the definition of the grasp matrix in body frame (6), we need to consider the contact model. In the case of planar hard fingers we can admit that the hand can exert only forces along the axes $x$ and $y$, without moments. In other words, the vectors describing the interaction at the contact is composed by two elements only. This implies that, to transform the local interaction in a complete wrench, for each contact we need a matrix

$$
B=\left[\begin{array}{llllll}
1 & 0 & 0 & 0 & 0 & 0 \\
0 & 1 & 0 & 0 & 0 & 0
\end{array}\right]^{T}
$$




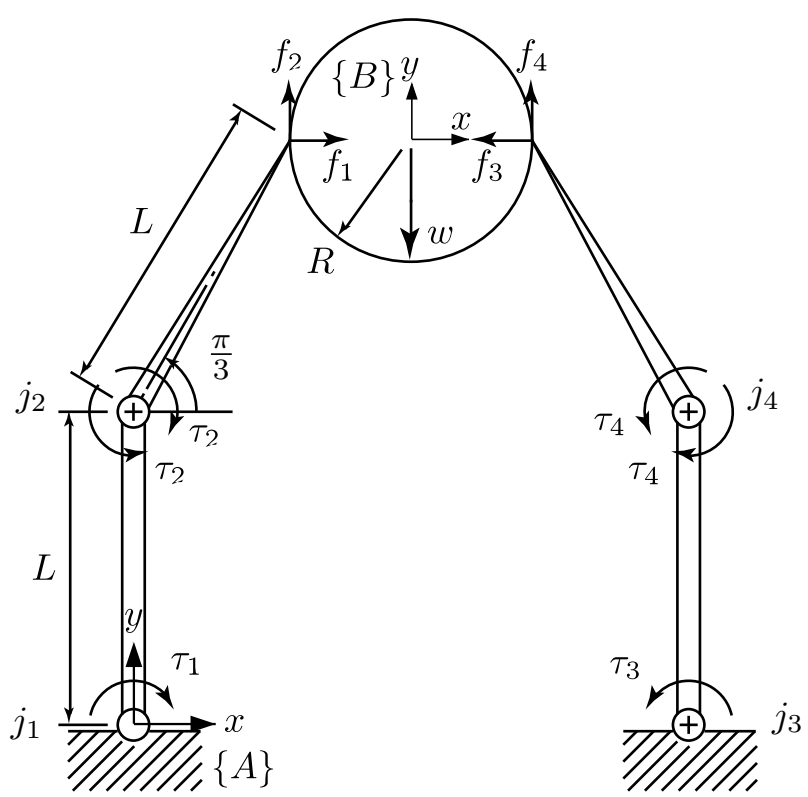

Figure 3: Grasp of a circular object by a two-fingered robotic hand.

The co-adjoint matrix in (6), by its definition in (91), can be computed considering the rotation matrices

$$
R_{b c_{1}^{o}}=R_{b c_{2}^{o}}=I_{3},
$$

where $I_{3}$ is an identity matrix of dimension 3 , and the vectors

$$
d_{b c_{1}^{o}}=\left[\begin{array}{c}
-R \\
0 \\
0
\end{array}\right], \quad d_{b c_{2}^{o}}=\left[\begin{array}{c}
R \\
0 \\
0
\end{array}\right],
$$

describing the position of the object contact frames $\left\{C_{1}^{o}\right\}$ and $\left\{C_{2}^{o}\right\}$, respectively, in frame $\{B\}$. Selecting only the components of the grasp matrix describing actions lying on the plane, the grasp matrix in body frame for the parameters values we choose, finally appears as

$$
{ }^{b} G=\left[\begin{array}{rrrr}
1 & 0 & 1 & 0 \\
0 & 1 & 0 & 1 \\
0 & -1 & 0 & 1
\end{array}\right] .
$$

In order to compute the hand Jacobian, we basically need to compute the spatial Jacobian matrices of the two contact frames, indicated as ${ }^{a} J_{1}$ and ${ }^{a} J_{2}$ respectively. Considering the vector $\omega=[0,0,1]^{T}$ of the joint axes, and the geometry of the system, from (102) we obtain the twists

$$
\xi_{1}=\left[\begin{array}{l}
0 \\
0 \\
0 \\
0 \\
0 \\
1
\end{array}\right], \quad \xi_{2}=\left[\begin{array}{l}
L \\
0 \\
0 \\
0 \\
0 \\
1
\end{array}\right], \quad \xi_{3}=\left[\begin{array}{c}
0 \\
-L-2 R \\
0 \\
0 \\
0 \\
1
\end{array}\right], \quad \xi_{4}=\left[\begin{array}{c}
L \\
-L-2 R \\
0 \\
0 \\
0 \\
1
\end{array}\right] .
$$

Setting to zero the value of the joint parameters $q$ in the reference configuration of Figure 3, from (101) it follows that $\xi_{2}^{\prime}=\xi_{2}$ and $\xi_{4}^{\prime}=\xi_{4}$. Finally, from the transformation in (17), for the geometric parameters 
that we are using, the hand Jacobian matrix in object contact frames results in

$$
c^{o} J=\left[\begin{array}{cccc}
-1.866 & -0.866 & 0 & 0 \\
0.500 & 0.500 & 0 & 0 \\
0 & 0 & -1.866 & -0.866 \\
0 & 0 & -0.500 & -0.500
\end{array}\right] .
$$

The derivatives of the Jacobian matrix, defined in (22), can be easily computed by (111) and (120), obtaining

$$
Q=\left[\begin{array}{llll}
0 & 0 & 0 & 0 \\
1 & 0 & 0 & 0 \\
0 & 0 & 0 & 0 \\
0 & 0 & 1 & 0
\end{array}\right], \quad U=\left[\begin{array}{ccc}
1 & -1 & 3.366 \\
1 & -1 & 2.366 \\
1 & 1 & 3.366 \\
1 & 1 & 2.366
\end{array}\right]
$$

\subsubsection{Perturbed Configuration for Fully Actuated Hand}

The aim here is to characterize some of the structural properties of the fully actuated grasp. Therefore, we perform the decomposition of the nullspace matrix as in (56). All the above definitions hold defining a synergy matrix $S=I_{\sharp q}$, or equivalently using the Fundamental Grasp Matrix for a fully actuated hand in (121). In this case, all the previous definitions given in Section 5 can be recovered, with the only exception of the external structural forces, where we have to impose the nullity of the hand level variables $\delta q$ and $\delta \tau$, instead of $\delta \sigma_{r}$ and $\delta \eta$. In this example, the nullspace of the FGM has dimension $\sharp w+\sharp q=7$. Within this 7-dimensional subspace the subspace of kinematic grasp displacements has dimension three, the pure squeeze subspace has dimension one: together they complete the subspace of internal system perturbations (no spurious squeeze and redundant motion of the hand are present). The external structural force subspace has null dimension, meaning that every external perturbation needs a counterpart on the hand actuation to preserve the equilibrium of the system. For the kinematic grasp displacements, a finite displacement of the object $\delta u_{x}=0.001$ in the $x$ direction, as in Figure 4(a), is possible with the corresponding variations in joint torques and angles

$$
\begin{gathered}
\delta \tau=\left[\begin{array}{llll}
0.001 & 0 & 0.001 & 0
\end{array}\right]^{T}, \\
\delta q=\left[\begin{array}{llll}
-0.001 & 0.001 & -0.001 & 0.001
\end{array}\right]^{T} .
\end{gathered}
$$

For an object displacement $\delta u_{y}=-0.001$, Figure 4(b), the corresponding variations in the joint torques and joint angles are

$$
\begin{aligned}
\delta \tau & =\left[\begin{array}{llll}
-0.0010 & -0.0027 & 0.0010 & 0.0027
\end{array}\right]^{T}, \\
\delta q & =\left[\begin{array}{llll}
0.0017 & -0.0037 & -0.0017 & 0.0037
\end{array}\right]^{T} .
\end{aligned}
$$

For an object rotation $\delta u_{\alpha}=0.001$, Figure 4(c), the variations in the joint torques and joint angles are then

$$
\begin{gathered}
\delta \tau=\left[\begin{array}{llll}
0.0033 & 0.0040 & 0.0033 & 0.0040
\end{array}\right]^{T}, \\
\delta q=\left[\begin{array}{llll}
0.0017 & -0.0037 & 0.0017 & -0.0037
\end{array}\right]^{T} .
\end{gathered}
$$

Finally, the variations of joint torques and joint angles for the squeeze, represented in Figure 4(d), are

$$
\begin{aligned}
& \delta \tau=\left[\begin{array}{llll}
-0.0018 & -0.0010 & 0.0018 & 0.0010
\end{array}\right]^{T}, \\
& \delta q=\left[\begin{array}{llll}
-0.0001 & 0.0001 & 0.0001 & -0.0001
\end{array}\right]^{T} .
\end{aligned}
$$




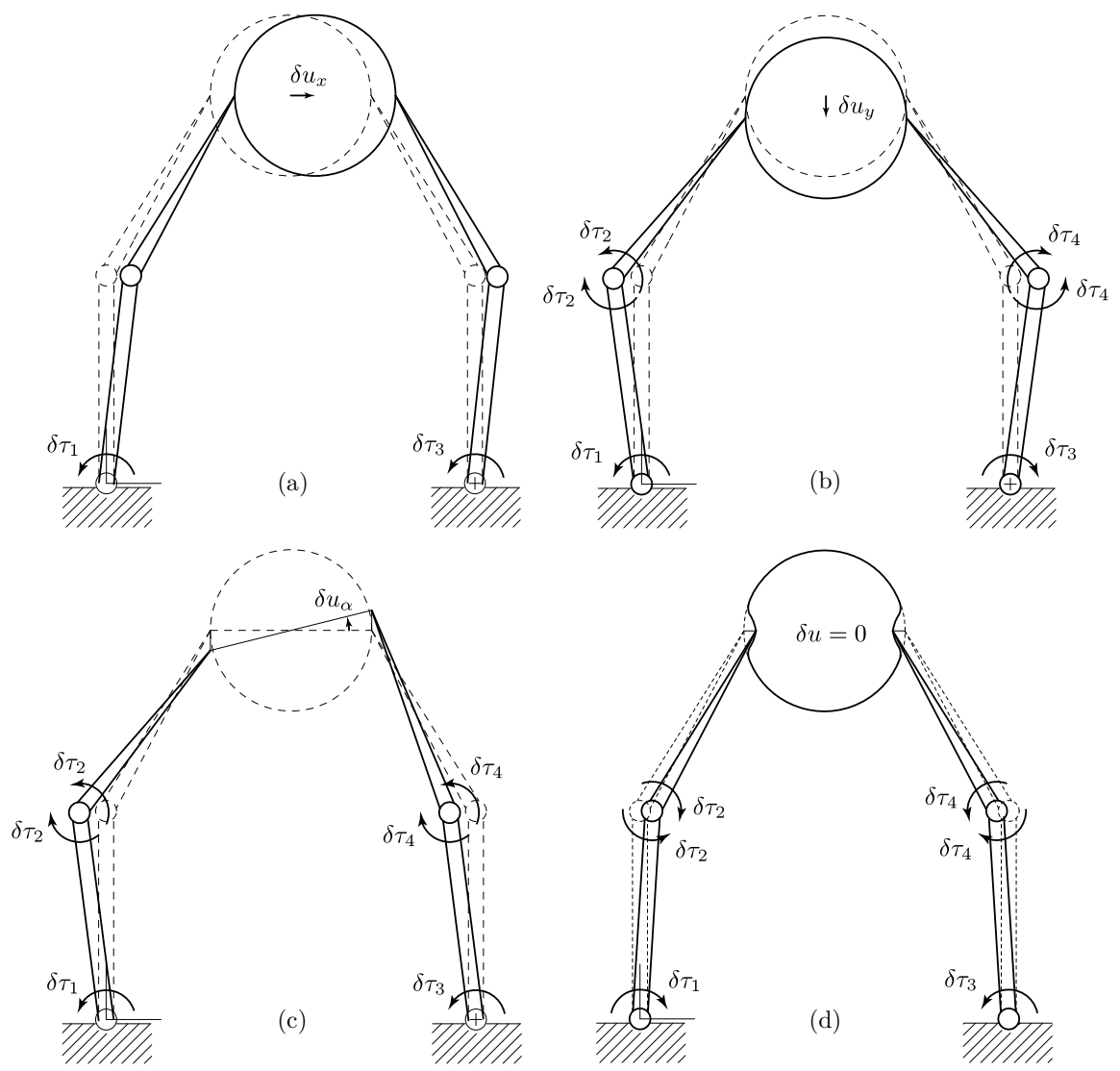

Figure 4: Graphic representations of numerical results for the internal precision grasp variations. The Figures (a-c) represent the kinematic grasp displacements, i.e. the object movements that the hand can impose to the object without modifying the contact forces. The Figure (d) represent a pure squeeze, i.e. a contact force variation obtained without moving the grasped object.

\subsubsection{A Synergy in the Precision Grasp}

Having inspected the properties of the fully-actuated system, we can then evaluate the effect of underactuation on the system properties by introducing only one synergy. The synergies proposed both here and later in section 9.2.2, are generated (by a reverse-engineering process) after the nullspace decomposition for the fully-actuated system with elastic joints has been carried out, by properly combining some solutions in order to synthesize a desired task. GEROME-B or Find-X can be profitably employed here to verify the results.

Introducing a single column synergy matrix as follows

$$
S=\left[\begin{array}{llll}
-0.2866 & 0.0034 & 0.2866 & -0.0034
\end{array}\right]^{T},
$$

we can write the FGM introduced in (43), for the synergistic underactuated case. Decomposing the nullspace we can verify that are left one pure squeeze, and three external forces and displacements, which together complete the basis of the nullspace. To elicit the nature of this synergy, we can evaluate the system response to a predictable input. To this aim, we can use the Find-X method or GEROMEB, obtaining, of course, the same results. Since the pure squeeze still exists for this system, with the above synergy it is always possible for a certain $\delta \sigma_{r}$ to keep the object in its starting configuration 
$(\delta u=0)$ for a null external perturbation $\delta w_{e}^{b}=[0,0,0]^{T}$. In fact, by applying $\delta \sigma_{r}=1$, we get $\delta f_{c}=[0.9039,0,-0.9039,0]$ with $\delta u=[0,0,0]^{T}$. This because $S$ was defined as proportional to the (single) column of the pure squeeze found for for the fully-actuated system.

\subsection{Power Grasp}

As a second test case, we consider a square of side $2 L$ grasped by a spider-like hand composed by two fingers, and a total of 8 joints $\left[j_{1}, \ldots, j_{8}\right]$. The notation is similar to the previous example. Figure 5

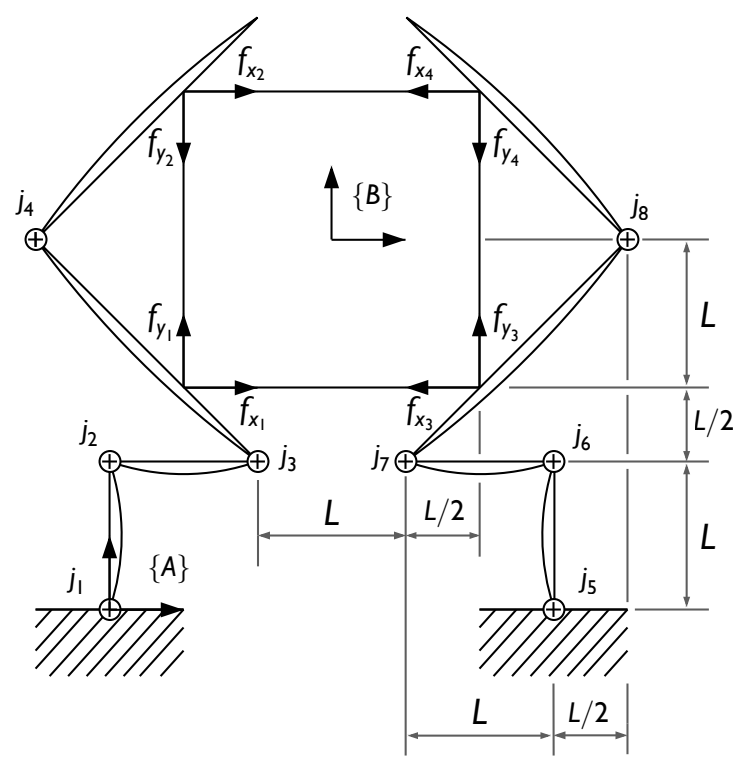

Figure 5: Grasp of a square by a two fingered spider-like hand.

shows the starting configuration of the hand and the initial contact preload. All the force vectors have unit lengths and the directions depicted. We consider every contact frame initially parallel to the frame $\{A\}$. The contact points, with respect to the frame $\{B\}$, are placed respectively in

$$
d_{b c_{1}^{o}}=\left[\begin{array}{c}
-L \\
-L \\
0
\end{array}\right], \quad d_{b c_{2}^{o}}=\left[\begin{array}{c}
-L \\
L \\
0
\end{array}\right], \quad d_{b c_{3}^{o}}=\left[\begin{array}{c}
L \\
-L \\
0
\end{array}\right], \quad d_{b c_{4}^{o}}=\left[\begin{array}{c}
L \\
L \\
0
\end{array}\right] .
$$

As in the previous example, we consider the numerical values $L=1 \mathrm{~m}, k_{q}=10 \mathrm{~N} / \mathrm{rad}, k_{c}=10 \mathrm{~N} / \mathrm{m}$, $k_{s}=10 \mathrm{~N} / \mathrm{m}$. For hard finger-like contacts, for which we can use (63), the grasp matrix in body frame results in

$$
{ }^{b} G=\left[\begin{array}{cccccccc}
1 & 0 & 1 & 0 & 1 & 0 & 1 & 0 \\
0 & 1 & 0 & 1 & 0 & 1 & 0 & 1 \\
1 & -1 & -1 & -1 & 1 & 1 & -1 & 1
\end{array}\right] .
$$

From the geometry in Figure 5, considering that every joint has $\omega=[0,0,1]^{T}$, we obtain the twists

$$
\xi_{1}=\left[\begin{array}{l}
0 \\
0 \\
0 \\
0 \\
0 \\
1
\end{array}\right], \quad \xi_{2}=\left[\begin{array}{l}
L \\
0 \\
0 \\
0 \\
0 \\
1
\end{array}\right], \quad \xi_{3}=\left[\begin{array}{c}
L \\
-L \\
0 \\
0 \\
0 \\
1
\end{array}\right], \quad \xi_{4}=\left[\begin{array}{c}
(5 / 2) L \\
(1 / 2) L \\
0 \\
0 \\
0 \\
1
\end{array}\right]
$$




$$
\xi_{5}=\left[\begin{array}{c}
0 \\
-3 L \\
0 \\
0 \\
0 \\
1
\end{array}\right], \quad \xi_{6}=\left[\begin{array}{c}
L \\
-3 L \\
0 \\
0 \\
0 \\
1
\end{array}\right], \quad \xi_{7}=\left[\begin{array}{c}
L \\
-2 L \\
0 \\
0 \\
0 \\
1
\end{array}\right], \quad \xi_{8}=\left[\begin{array}{c}
(5 / 2) L \\
-(7 / 2) L \\
0 \\
0 \\
0 \\
1
\end{array}\right] .
$$

From (77), considering a null value for the joint parameters $q$ in the reference configuration, according to (17), the hand Jacobian results in

$$
c^{o} J=\left[\begin{array}{cccccccc}
-1.500 & -0.500 & -0.500 & 0 & 0 & 0 & 0 & 0 \\
0.500 & 0.500 & -0.500 & 0 & 0 & 0 & 0 & 0 \\
-3.500 & -2.500 & -2.500 & -1 & 0 & 0 & 0 & 0 \\
0.500 & 0.500 & -0.500 & 1 & 0 & 0 & 0 & 0 \\
0 & 0 & 0 & 0 & -1.500 & -0.500 & -0.500 & 0 \\
0 & 0 & 0 & 0 & -0.500 & -0.500 & 0.500 & 0 \\
0 & 0 & 0 & 0 & -3.500 & -2.500 & -2.500 & -1 \\
0 & 0 & 0 & 0 & -0.500 & -0.500 & 0.500 & -1
\end{array}\right] .
$$

The derivatives of the Jacobian matrix, computed by the rules (111) and (120), appear as follows

$$
Q=\left[\begin{array}{cccccccc}
0 & 0 & 0 & 0 & 0 & 0 & 0 & 0 \\
0 & 0 & 0 & 0 & 0 & 0 & 0 & 0 \\
2 & 2 & 0 & 0 & 0 & 0 & 0 & 0 \\
3 & -2 & -3 & 0 & 0 & 0 & 0 & 0 \\
0 & 0 & 0 & 0 & 0 & 0 & 0 & 0 \\
0 & 0 & 0 & 0 & 0 & 0 & 0 & 0 \\
0 & 0 & 0 & 0 & 2 & 2 & 0 & 0 \\
0 & 0 & 0 & 0 & -3 & -2 & -3 & 0
\end{array}\right], \quad U=\left[\begin{array}{ccc}
0 & -2 & 3 \\
0 & -2 & 3 \\
0 & -2 & 1 \\
-1 & -1 & 2 \\
0 & 2 & 3 \\
0 & 2 & 3 \\
0 & 2 & 1 \\
-1 & 1 & 2
\end{array}\right] .
$$

\subsubsection{Perturbed Configuration for Fully Actuated Hand}

By employing the matrices presented in the previous section, we can build the FGM for the fully actuated case as in (121). In this case we obtain that $A \in \mathbb{R}^{43 \times 54}$ and, consequently, the basis for the $N(A)$ is $\Gamma \in \mathbb{R}^{54 \times 11}$. Performing the decomposition of the nullbasis $\Gamma$, as in (56), we obtain that the kinematic grasp subspace has dimension three, the pure squeeze subspace has dimension five, and together they complete the subspace of internal forces and displacements. Conversely, the subspace of external forces and displacements has dimension three. For the kinematic grasp displacements, simulation results show that it is possible to have a finite displacement of the object $\delta u_{x}=0.001$, as in Figure 6(a), with no torque variations, but with a joint angle displacement of

$$
\delta q=10^{-3}\left[\begin{array}{llllllll}
-1 & 1 & 0 & 0 & -1 & 1 & 0 & 0
\end{array}\right]^{T} .
$$

For $\delta u_{y}=0.001$, the corresponding variations in the joint torques and joint angles are

$$
\begin{gathered}
\delta \tau=10^{-3}\left[\begin{array}{llllllll}
-2 & -2 & 0 & 0 & 2 & 2 & 0 & 0
\end{array}\right]^{T} \\
\delta q=10^{-3}\left[\begin{array}{llllllll}
0 & 1 & -1 & 0 & 0 & -1 & 1 & 0
\end{array}\right]^{T},
\end{gathered}
$$

with pictorial representations sketched in Figure 6(b). For an object rotation $\delta u_{\alpha}=0.001$, Figure 6(c), the variations in the joint torques and joint angles are then

$$
\begin{gathered}
\delta \tau=10^{-3}\left[\begin{array}{cccccccc}
3 & 3 & 0 & 0 & 3 & 3 & 0 & 0
\end{array}\right]^{T}, \\
\delta q=10^{-3}\left[\begin{array}{lllllll}
-1.5 & 1 & 1.5 & 0 & -1.5 & 1 & 1.5
\end{array}\right]^{T} .
\end{gathered}
$$



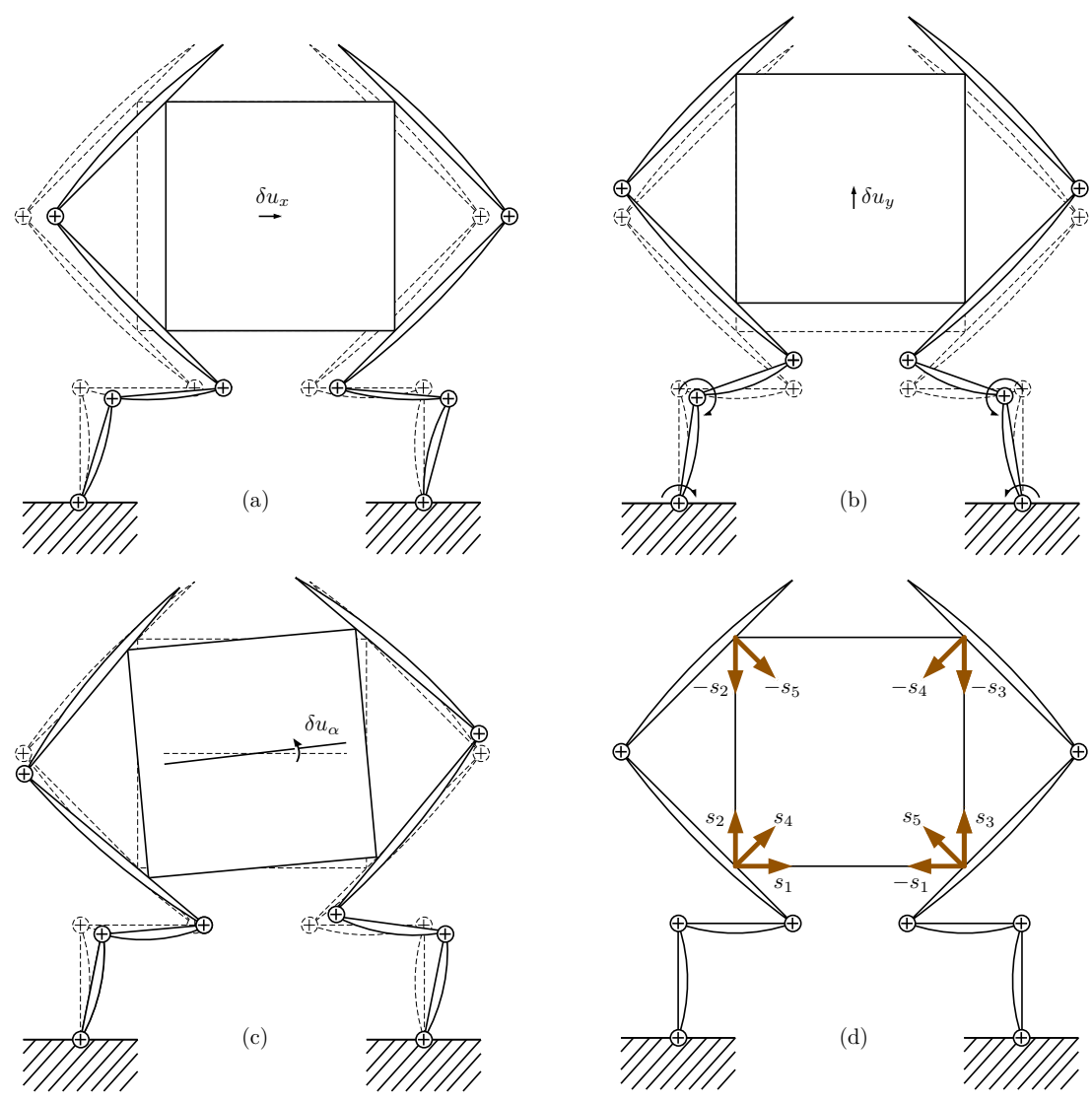

Figure 6: Graphic representations of numerical results for the internal power grasp variations. The Figures (a-c) represent the kinematic grasp displacements, i.e. the object movements that the hand can impose to the object without modifying the contact forces. The Figure (d) represent the pure squeeze. In this case the two diagonals of the square and three of the four sides (left, bottom and right one) of the object can be individually squeezed, without moving the object. This is not possible for the top side one.

The five possibilities for the pure squeeze are sketched in Figure 6(d), where the $i^{\text {th }}$ couple of forces $s_{i}$ and $-s_{i}$ correspond to the $i^{\text {th }}$ achievable squeeze.

\subsubsection{A Synergy in the Power Grasp}

Underactuating the hand with the following single-column synergy matrix

$$
\left.S=\begin{array}{cccc}
{[-0.6500} & 0 & -0.3200 & -0.4000 \\
0.6500 & 0 & 0.3200 & 0.4000
\end{array}\right]^{T},
$$

we get one possibility for the squeeze and three external solutions. To unveil the properties of this synergy we can study how the system responds to different inputs. In the absence of an external interaction, $\delta w_{e}^{b}=0$, and with an unitary synergistic actuation, $\delta \sigma_{r}=1$, contact forces and object displacements become

$$
\begin{gathered}
\left.\delta f_{c^{h}}^{c^{o}}=\begin{array}{cccc}
0.5043 & 0.5043 & 0.5043 & -0.5043 \\
-0.5043 & 0.5043 & -0.5043 & -0.5043
\end{array}\right]^{T}, \\
\delta u=\left[\begin{array}{lll}
0 & 0 & 0
\end{array}\right]^{T},
\end{gathered}
$$


indicating that we are purely squeezing the object along both diagonals. It is worth noting that the above synergy was constructed by summing two columns of the pure squeeze relative to the fully-actuated system.

\section{CONCLUSIONS}

In this work, we presented a framework to model and study the structural properties of a grasp, performed by a general robotic hand, in a quasi-static setting. Synergistic underactuation is included in the analysis, as well as compliant characteristics at various levels, in the fingerpads and in the actuation system, both for joints and synergies. The mathematical model presented considers also the derivatives of the Jacobian matrix and employs an object-centric formulation of the grasp matrix, since other definitions may lead to potentially misleading conclusions. Considering all the system equations, the Fundamental Grasp Equation and the Fundamental Grasp Matrix are defined. An extension of the Gauss-Jordan elimination method, called GEROME-B, able to act on block partitioned matrices, is presented. Thanks to this, the Fundamental Grasp Matrix in canonical form is found, corresponding to a new set of equations where each dependent variable of the system is a function only of the independent ones. Main relevant properties of the grasp can be discovered in this form, as a basis for the controllable internal forces and the grasp compliance. It is important to note that their expressions can be found even in symbolic form, as a functions of the basic matrices of the system, such as the Jacobian matrix, the grasp matrix, and so on. As GEROME-B acts in symbolic form, another method, called Find-X, is presented as a numerical efficient counterpart to find input-output relationships between the variables of the system.

Other more specific categories of system perturbations are later defined, in terms of nullity or nonnullity of some variables, such as the pure squeeze, when the hand acts on the object without changing its configuration, or kinematic object displacements, when the object is rigidly moved. An algorithm based on the extensive employment of the reduced row echelon form (RREF) has been presented, that allows to discover them inside the solution space of the system, and even to find the particular inputs that the system needs to perform them.

The search of feasible solutions was further discussed, considering all the equations of the system as constraints for the feasible (non-)nullity patterns of variables, until arriving to a complete taxonomy of the grasp performed by synergistic underactuated hands. In order to assess the validity of the proposed method, some numerical tests have been presented for two different grasps: a precision grasp and a power grasp. These demonstrated (i) the importance of the terms originated by the derivatives of the Jacobian matrix in the presence of a preload contact force to obtain physically meaningful results, and (ii) the influence of the synergistic actuation on the structural properties of a grasp configuration.

\section{ACKNOWLEDGMENTS}

This work is supported by the European Commission under the CP-IP grant no. 248587 "THE Hand Embodied", within the FP7-2007-2013 program "Cognitive Systems and Robotics", ERC Advanced Grant no. 291166 "SoftHands" - A Theory of Soft Synergies for a New Generation of Artificial Hands and by the grant no. 600918 "PaCMan" - Probabilistic and Compositional Representations of Objects for Robotic Manipulation - within the FP7-ICT-2011-9 program "Cognitive Systems".

\section{A HOMOGENEOUS TRANSFORMATIONS AND ADJOINT OPERATORS}

Given a point $p \in \mathbb{R}^{3}$, we define its homogeneous form as

$$
\bar{p}=\left[\begin{array}{l}
p \\
1
\end{array}\right] .
$$

Considering two reference frames $\{A\}=\left\{O_{a} ; x_{a}, y_{a}, z_{a}\right\}$ and $\{B\}=\left\{O_{b} ; x_{b}, y_{b}, z_{b}\right\}$, let $d_{a b} \in \mathbb{R}^{3}$ be the vector going from $O_{a}$ to $O_{b}$, and $R_{a b} \in \mathbb{R}^{3 \times 3}$ be the rotation matrix describing the configuration of 
$\{B\}$ with respect to $\{A\}$. The descriptions of the point $p$ in the two reference frames are related by the expression

$$
\bar{p}_{a}=\left[\begin{array}{cc}
R_{a b} & d_{a b} \\
0 & 1
\end{array}\right] \bar{p}_{b}:=g_{a b} \bar{p}_{b}
$$

where we implicitly defined the homogeneous transformation matrix as

$$
g_{a b}=\left[\begin{array}{cc}
R_{a b} & d_{a b} \\
0 & 1
\end{array}\right] .
$$

With the symbol $\bullet$ we indicate the cross product matrix, thus such that, for $x, y \in \mathbb{R}^{3}$, it holds

$$
\hat{x} y=x \times y .
$$

The adjoint matrix and the co-adjoint matrix, which properties are explained in Section 2, have the following expressions

$$
\begin{aligned}
\operatorname{Ad}_{g_{a b}} & =\left[\begin{array}{cc}
R_{a b} & \hat{d}_{a b} R_{a b} \\
0 & R_{a b}
\end{array}\right], \\
\operatorname{Ad}_{g_{a b}}^{-T} & =\left[\begin{array}{cc}
R_{a b} & 0 \\
\hat{d}_{a b} R_{a b} & R_{a b}
\end{array}\right] .
\end{aligned}
$$

Introducing the operator $(\bullet)^{\swarrow}$ such that $(\hat{x})^{\check{L}}=x$, by direct calculation, from (89) it follows that

$$
x \times y=(\hat{x} \hat{y}-\hat{y} \hat{x}) .
$$

Expanding the definition of the operator $\hat{\bullet}$ to act on twists $\xi=\left[v^{T}, \omega^{T}\right]^{T} \in \operatorname{se}(3)$ as

$$
\hat{\xi}=\left[\begin{array}{ll}
\hat{\omega} & v \\
0 & 0
\end{array}\right]
$$

in analogy with (92), we define the Lie bracket as an operator acting on two elements of se(3) according with the rule

$$
\left[\xi_{1} ; \xi_{2}\right]=\left(\hat{\xi}_{1} \hat{\xi}_{2}-\hat{\xi}_{2} \hat{\xi}_{1}\right)
$$

It is worth observing that the result of the Lie bracket of two twists it is also a twist. The operation described in (94), can be realized by a proper multiplication operation in the form

$$
\left[\xi_{1} ; \xi_{2}\right]=\left[\begin{array}{cc}
\hat{\omega}_{1} & \hat{v}_{1} \\
0 & \hat{\omega}_{1}
\end{array}\right]\left[\begin{array}{c}
v_{2} \\
\omega_{2}
\end{array}\right]:=\operatorname{ad}\left(\xi_{1}\right) \xi_{2},
$$

where we introduced the adjoint matrix (in lower case), also called Lie adjoint hereafter, as

$$
\operatorname{ad}(\xi)=\left[\begin{array}{cc}
\hat{\omega} & \hat{v} \\
0 & \hat{\omega}
\end{array}\right]
$$

For later use, we show that between the adjoint matrix (Ad) and the Lie adjoint (ad), the following relationships hold

$$
\begin{gathered}
\operatorname{Ad}_{e^{\hat{\xi}}}=e^{\operatorname{ad}(\xi)}, \\
\operatorname{Ad}_{e^{\hat{\xi}_{1}}} e^{\operatorname{ad}\left(\xi_{2}\right)} \operatorname{Ad}_{e^{\hat{\xi}_{1}}}^{-1}=\operatorname{ad}\left(\operatorname{Ad}_{e^{\hat{\xi}_{1}}} \xi_{2}\right) .
\end{gathered}
$$

More details about the exponential of a twist are presented in the next section. 


\section{B SPATIAL JACOBIAN MATRIX}

For the reader convenience, we briefly present how to compute the spatial Jacobian matrix of serial manipulator, that is a matrix ${ }^{s} J(q) \in \mathbb{R}^{6 \times \sharp q}$ such that

$$
\xi_{a e}^{a}={ }^{s} J(q) \dot{q},
$$

where the vector $\xi_{a e}^{a} \in \mathbb{R}^{6}$ is the twist, with components in $\{A\}$, of a frame of reference that is instantly superimposed to the absolute frame, but moves rigidly with the movement of the end-effector. It is useful to remind that the Jacobian matrix can be written as

$$
{ }^{a} J(q)=\left[\begin{array}{llll}
\xi_{1} & \xi_{2}^{\prime} & \ldots & \xi_{n}^{\prime}
\end{array}\right]
$$

where

$$
\xi_{k}^{\prime}=\operatorname{Ad}_{\left(e^{\hat{\xi}_{1} q_{1} \ldots e^{\hat{\xi}_{k-1} q_{k-1}}}\right)} \xi_{k} .
$$

It is worth noting that the terms $\xi_{k}$ in (101) correspond to the the $k^{\text {th }}$ joint twist, and $\xi_{k}^{\prime}$ are their transformation imposed by the robot configuration. The twists for a prismatic and a revolute joint have the forms

$$
\xi_{p}=\left[\begin{array}{c}
v_{p} \\
0
\end{array}\right], \quad \xi_{r}=\left[\begin{array}{c}
-\omega_{r} \times p_{r} \\
\omega_{r}
\end{array}\right]=\left[\begin{array}{c}
v_{r} \\
\omega_{r}
\end{array}\right],
$$

where $v_{p} \in \mathbb{R}^{3}$ is a unit vector pointing in direction of the axis of the prismatic joint, $\omega_{r} \in \mathbb{R}^{3}$ is a unit vector identifying the axis of the revolute joint and $p_{r}$ is a point on this axis. It is possible to demonstrate that the exponential of the twists in (102), necessary to compute (101) and (100), are given by

$$
e^{\hat{\xi}_{p} q_{p}}=\left[\begin{array}{cc}
I & v_{p} q_{p} \\
0 & 1
\end{array}\right], \quad e^{\hat{\xi}_{r} q_{r}}=\left[\begin{array}{cc}
e^{\hat{\omega}_{r} q_{r}} & \left(I-e^{\hat{\omega}_{r} q_{r}}\right)\left(\omega_{r} \times v_{r}\right)+\omega_{r} \omega_{r}^{T} v_{r} q_{r} \\
0 & 1
\end{array}\right],
$$

where

$$
e^{\hat{\omega}_{r} q_{r}}=I+\hat{\omega}_{r} \sin q_{r}+\hat{\omega}_{r}^{2}\left(1-\cos q_{r}\right) .
$$

Equation (104) corresponds to a rotation of an angle $q_{r}$ around an axis identified by the vector $\omega_{r}$, and it is known as Rodrigues' formula.

\section{DERIVATIVE TERMS OF THE JACOBIAN MATRIX}

C.1 Derivative of the Jacobian matrix with respect to the hand configuration

The definitions given in Appendices A and B are useful now to calculate the derivatives of the Jacobian matrix, seen in (22). Other details about the results provided in this section can be found in [Murray et al., 1994, Hall, 2003] and in [Selig, 2005]. For the seek of simplicity, but without loss of generality, in this section we will find the derivatives of a Jacobian matrix of a serial robot, such, for example, a finger of the hand. The reader will easily extend the results to the whole hand Jacobian matrix.

Let's consider first the derivative with respect to the hand configuration, that is the variable $q$, of the Jacobian matrix describing the twist of the $i^{\text {th }}$ contact frame, as defined in (17). In other words we are looking for a direct calculation method for

$$
Q_{i}=\frac{\partial{ }^{c_{i}^{o}} J_{i}^{T}(q, u) f_{c_{i}^{h}}^{c_{i}^{o}}}{\partial q}
$$

or equivalently

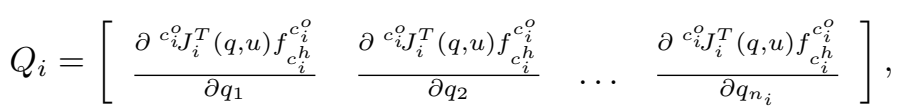


where $n_{i}$ is the number of joints affecting the motion of the contact frame. From (17) it follows that the $j^{\text {th }}$ term in (106) can be even expressed as

$$
\frac{\partial\left(B_{i}^{T} \operatorname{Ad}_{g_{c_{i}^{o} b}} \operatorname{Ad}_{g_{b a}(u)}{ }^{a} J_{i}(q)\right)^{T} f_{c_{i}^{h}}^{c_{i}^{o}}}{\partial q_{j}}=\left(\frac{\partial^{a} J_{i}(q)}{\partial q_{j}}\right)^{T} \operatorname{Ad}_{g_{c_{i}^{o} a}(u)}^{T} B_{i} f_{c_{i}^{h}}^{c_{i}^{o}},
$$

where we used the fact that the operations of transposition and derivative with respect to a scalar quantity commute. Considering (107), the problem is now reduced to find the derivative of the spatial Jacobian for a serial robot, as in (100).

It is possible to approach the problem searching the derivative of the twists composing it, that is

$$
\begin{aligned}
\frac{\partial \xi_{k}^{\prime}}{\partial q_{j}}=\frac{\partial}{\partial q_{j}} & \left(\operatorname{Ad}_{\left(e^{\hat{\xi}_{1} q_{1} \ldots e^{\hat{\xi}_{k-1} q_{k-1}}}\right)} \xi_{k}\right)= \\
= & \frac{\partial}{\partial q_{j}}\left(\operatorname{Ad}_{e^{\hat{\xi}_{1} q_{1}}} \ldots \operatorname{Ad}_{e^{\hat{\xi}_{j} q_{j}}} \ldots \operatorname{Ad}_{e^{\hat{\xi}_{k-1} q_{k-1}}} \xi_{k}\right) .
\end{aligned}
$$

Remembering the property in (97), from (108) it directly follows that

$$
\begin{aligned}
\frac{\partial \xi_{k}^{\prime}}{\partial q_{j}}=\operatorname{Ad}_{e^{\hat{\xi}_{1} q_{1}}} \ldots \frac{\partial e^{\operatorname{ad}\left(\xi_{j}\right) q_{j}}}{\partial q_{j}} \ldots \operatorname{Ad}_{e^{\hat{\xi}_{k-1} q_{k-1}}} \xi_{k}= \\
=\operatorname{Ad}_{e^{\hat{\xi}_{1} q_{1}}} \ldots \operatorname{ad}\left(\xi_{j}\right) e^{\operatorname{ad}\left(\xi_{j}\right) q_{j}} \ldots \operatorname{Ad}_{e^{\hat{\xi}_{k-1} q_{k-1}}} \xi_{k},
\end{aligned}
$$

where the last term can be easily rewritten as

$$
\begin{aligned}
& \operatorname{Ad}_{e^{\hat{\xi}_{1} q_{1}}} \ldots \operatorname{Ad}_{e^{\hat{\xi}_{j-1} q_{j-1}}} \operatorname{ad}\left(\xi_{j}\right) \operatorname{Ad}_{e^{-\hat{\xi}_{j-1} q_{j-1}}} \ldots \\
& \quad \ldots \operatorname{Ad}_{e^{-\hat{\xi}_{1} q_{1}}} \operatorname{Ad}_{e^{\hat{\xi}_{1} q_{1}}} \ldots \operatorname{Ad}_{e^{\hat{\xi}_{k-1} q_{k-1}}} \xi_{k}=\operatorname{ad}\left(\operatorname{Ad}_{\left(e^{\hat{\xi}_{1} q_{1} \ldots e^{\hat{\xi}_{j-1} q_{j-1}}}\right)} \xi_{j}\right) \xi_{k}^{\prime}=\operatorname{ad}\left(\xi_{j}^{\prime}\right) \xi_{k}^{\prime} .
\end{aligned}
$$

The results can be finally summarized as

$$
\frac{\partial \xi_{k}^{\prime}}{\partial q_{j}}= \begin{cases}\operatorname{ad}\left(\xi_{j}^{\prime}\right) \xi_{k}^{\prime} & \text { if } j<k \\ 0 & \text { otherwise. }\end{cases}
$$

Thanks to (111), using the definition (96), it is now trivial to compute (107), thus also (105).

\section{C.2 Derivative of the Jacobian matrix with respect to the object configuration}

Let's consider now the problem of calculating the derivative of the Jacobian matrix of the $i^{\text {th }}$ contact frame with respect to the object configuration $u$, that is the term

$$
U_{i}=\frac{\partial c_{i}^{o} J_{i}^{T}(q, u) f_{c_{i}^{h}}^{c_{i}^{o}}}{\partial u} .
$$

As first step, considering that (112) can be equivalently written as

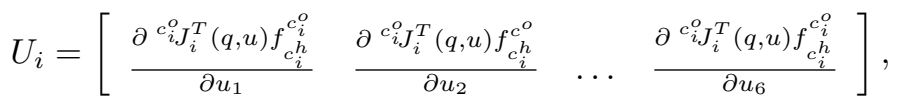

by virtue of (17), we reduce the problem to the calculation of

$$
\frac{\partial\left(B_{i}^{T} \operatorname{Ad}_{g_{c_{i} b}} \operatorname{Ad}_{g_{b a}(u)}{ }^{a} J_{i}(q)\right)^{T} f_{c_{i}^{h}}^{c_{i}^{o}}}{\partial u_{j}}={ }^{a} J_{i}(q)^{T}\left(\frac{\partial \operatorname{Ad}_{g_{b a}(u)}}{\partial u_{j}}\right)^{T} \operatorname{Ad}_{g_{c_{i}^{o} b}^{T}}^{T} B_{i} f_{c_{i}^{h}}^{c_{i}^{o}},
$$


that is finally

$$
\frac{\partial \operatorname{Ad}_{g_{b a}(u)}}{\partial u_{j}}=\frac{\partial \operatorname{Ad}_{g_{a b}^{-1}(u)}}{\partial u_{j}} .
$$

As seen in (13), a parametrization for the object twist is needed. We can obtain it introducing the Jacobian of a virtual kinematic chain described by a suitable Jacobian matrix of the form

$$
\begin{aligned}
& J_{o}(u)=\left[\begin{array}{llll}
\xi_{o_{1}} & \xi_{o_{2}}^{\prime} & \ldots & \xi_{o_{6}}^{\prime}
\end{array}\right], \\
& \xi_{o_{k}}^{\prime}=\operatorname{Ad}\left(e^{\hat{\xi}_{o_{1}} u_{1} \ldots e^{\hat{\xi}_{o_{k-1}} u_{k-1}}}\right) \xi_{o_{k}} .
\end{aligned}
$$

The same parametrization can be used to describe the homogeneous matrix $g_{a b}(u)$ as product of exponentials

$$
g_{a b}(u)=e^{\hat{\xi}_{1} u_{1}} e^{\hat{\xi}_{2} u_{2}} \ldots e^{\hat{\xi}_{6} u_{6}}
$$

Substituting (117) into (115), considering the properties of the adjoint matrix, it is easy to obtain

$$
\begin{aligned}
& \frac{\partial \operatorname{Ad}_{a b}-1(u)}{\partial u_{j}}=\frac{\partial}{\partial u_{j}} \operatorname{Ad}\left(e^{-\hat{\xi}_{o_{6}} u_{6}} e^{-\hat{\xi}_{o_{5}} u_{5}} \ldots e^{-\hat{\xi}_{o_{1}} u_{1}}\right)=
\end{aligned}
$$

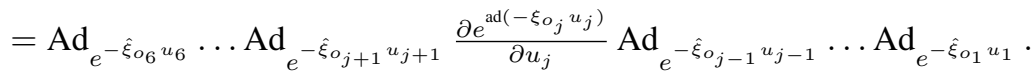

The derivative term in (118) can be also expressed as

$$
\begin{aligned}
& \frac{\partial e^{\operatorname{ad}\left(-\xi_{o_{j}} u_{j}\right)}}{\partial u_{j}}=\operatorname{ad}\left(-\xi_{o_{j}}\right) e^{\operatorname{ad}\left(-\xi_{o_{j}} u_{j}\right)}=\operatorname{ad}\left(-\xi_{o_{j}}\right) \operatorname{Ad}_{e}^{-\hat{\xi}_{o_{j} u_{j}}}= \\
& =\operatorname{Ad}_{e^{-\hat{\xi}_{o_{j} u_{j}}}} \ldots \mathrm{Ad}_{e^{-\hat{\xi}_{o_{1} u_{1}}}} \operatorname{Ad}_{e^{\hat{\xi}_{o_{1} u_{1}}}} \ldots \mathrm{Ad}_{e^{\hat{\xi}_{o_{j} u_{j}}}} \operatorname{ad}\left(-\xi_{o_{j}}\right) \mathrm{Ad}_{e^{-\hat{\xi}_{o_{j} u_{j}}}} .
\end{aligned}
$$

Substituting (119) in (118), considering the properties seen in (97) and in (98), we finally obtain

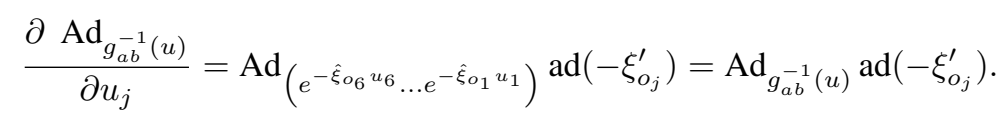

From (120), it is now trivial to compute (114), and thus (112).

\section{BLOCK ELEMENTS OF THE CANONICAL FORM OF THE FUNDAMENTAL GRASP MATRIX}

D.1 Fundamental Grasp Matrix for a Fully Actuated Hand Grasping an Object

The Fundamental Grasp Equation relative to the case of a grasp performed by a fully actuated hand can be obtained considering (8), (14), (19), (23), (25). This appears in the form

$$
\left[\begin{array}{ccccccc}
I_{\sharp f} & 0 & 0 & -K_{c} & K_{c} & 0 & 0 \\
-c^{o} \bar{J}^{T} & I_{\sharp \tau} & -\bar{U} & 0 & 0 & 0 & -\bar{Q} \\
0 & 0 & -{ }^{b} G K_{c}{ }^{b} \bar{G}_{t}^{T} & 0 & 0 & I_{\sharp w} & { }^{b} G K_{c} c^{o} \bar{J} \\
0 & 0 & 0 & I_{\sharp C^{h}} & 0 & 0 & -{ }^{o} \bar{J} \\
0 & 0 & -{ }^{b} \bar{G}_{t}^{T} & 0 & I_{\sharp C^{o}} & 0 & 0
\end{array}\right]\left[\begin{array}{c}
\delta f_{c^{h}}^{c^{o}} \\
\delta \tau \\
\delta u \\
\delta C_{a c^{c}}^{c^{o}} \\
\delta C_{a c^{\circ}}^{c^{\circ}} \\
\delta w_{e}^{b} \\
\delta q
\end{array}\right]=0 .
$$


Applying the algorithm GEROME-B to (121), this becomes in quasi-diagonal form as follows

$$
\left[\begin{array}{ccccccc}
I_{\sharp f} & 0 & 0 & 0 & 0 & W_{f}^{f} & R_{f}^{f} \\
0 & I_{\sharp \tau} & 0 & 0 & 0 & W_{\tau}^{f} & R_{\tau}^{f} \\
0 & 0 & I_{\sharp w} & 0 & 0 & W_{u}^{f} & R_{u}^{f} \\
0 & 0 & 0 & I_{\sharp C^{h}} & 0 & W_{C^{h}}^{f} & R_{C^{h}}^{f} \\
0 & 0 & 0 & 0 & I_{\sharp C^{o}} & W_{C^{o}}^{f} & R_{C^{\circ}}^{f}
\end{array}\right]\left[\begin{array}{c}
\delta f_{c^{\circ}}^{c^{o}} \\
\delta \tau \\
\delta u \\
\delta C_{a c^{o}}^{c^{h}} \\
\delta C_{a c^{\circ}}^{c^{\circ}} \\
\delta w_{e}^{b} \\
\delta q
\end{array}\right]=0 .
$$

The super-script $f$ was introduced to distinguish the blocks relative to the fully actuated case, from the cases with elastic joints and synergistic underactuation, respectively, presented later in this section. The blocks composing (122) have the following expression

$$
\begin{gathered}
W_{f}^{f}=K_{c}{ }^{b} \bar{G}_{t}^{T}\left({ }^{b} G K_{c}{ }^{b} \bar{G}_{t}^{T}\right)^{-1}, \\
W_{\tau}^{f}=\left({ }^{c} \bar{J}^{T} K_{c}{ }^{b} \bar{G}_{t}^{T}-\bar{U}\right)\left({ }^{b} G K_{c}{ }^{b} \bar{G}_{t}^{T}\right)^{-1}, \\
W_{u}^{f}=-\left({ }^{b} G K_{c}{ }^{b} \bar{G}_{t}^{T}\right)^{-1}, \\
W_{C^{h}}^{f}=0 \\
W_{C^{o}}^{f}=-{ }^{b} \bar{G}_{t}^{T}\left({ }^{b} G K_{c}{ }^{b} \bar{G}_{t}^{T}\right)^{-1}, \\
R_{f}^{f}=K_{c}\left({ }^{b} \bar{G}_{t}^{T}\left({ }^{b} G K_{c}^{b} \bar{G}_{t}^{T}\right)^{-1}{ }^{b} G K_{c}-I_{f}\right){ }^{o} \bar{J}, \\
R_{\tau}^{f}=-\bar{Q}-{ }^{c} \bar{J}^{T} K_{c}{ }^{c} \bar{J}+\left({ }^{c} \bar{J}^{T} K_{c} b \bar{G}_{t}^{T}-\bar{U}\right)\left({ }^{b} G K_{c}^{b} \bar{G}_{t}^{T}\right)^{-1}{ }^{b} G K_{c}{ }^{c} \bar{J}, \\
R_{u}^{f}=-\left({ }^{b} G K_{c}{ }^{b} \bar{G}_{t}^{T}\right)^{-1}{ }^{b} G K_{c}{ }^{c} \bar{J}, \\
R_{C^{h}}^{f}=-{ }^{c o} \bar{J}, \\
R_{C^{o}}^{f}=-{ }^{b} \bar{G}_{t}^{T}\left({ }^{b} G K_{c}{ }^{b} \bar{G}_{t}^{T}\right)^{-1}{ }^{b} G K_{c}{ }^{c} \bar{J} .
\end{gathered}
$$

D.2 Fundamental Grasp Matrix for a Fully Actuated Hand with Elastic Joints Grasping an Object

The Fundamental Grasp Equation describing the case of a hand with elastic joints can be obtained adding (27) to the system (122), resulting in

$$
\left[\begin{array}{cccccccc}
I_{\sharp f} & 0 & 0 & -K_{c} & K_{c} & 0 & 0 & 0 \\
-c^{o} \bar{J}^{T} & I_{\sharp \tau} & -\bar{U} & 0 & 0 & -\bar{Q} & 0 & 0 \\
0 & 0 & -{ }^{b} G K_{c}{ }^{b} \bar{G}_{t}^{T} & 0 & 0 & { }^{b} G K_{c}{ }^{c} \bar{J} & I_{\sharp w} & 0 \\
0 & 0 & 0 & I_{\sharp C h} & 0 & -c^{o} \bar{J} & 0 & 0 \\
0 & 0 & -{ }^{b} \bar{G}_{t}^{T} & 0 & I_{\sharp C^{o}} & 0 & 0 & 0 \\
0 & I_{\sharp \tau} & 0 & 0 & 0 & K_{q} & 0 & -K_{q}
\end{array}\right]\left[\begin{array}{c}
\delta f_{c^{h}}^{c^{o}} \\
\delta \tau \\
\delta u \\
\delta C_{a c^{o}}^{c^{o}} \\
\delta C_{a c^{o}}^{c^{c}} \\
\delta q \\
\delta w_{e}^{b} \\
\delta q_{r}
\end{array}\right]=0 .
$$


The canonical form is achieved applying GEROME-B to the coefficient matrix of (125). The final form of the system appears as

$$
\left[\begin{array}{cccccccc}
I_{\sharp f} & 0 & 0 & 0 & 0 & 0 & W_{f}^{e} & R_{f}^{e} \\
0 & I_{\sharp \tau} & 0 & 0 & 0 & 0 & W_{\tau}^{e} & R_{\tau}^{e} \\
0 & 0 & I_{\sharp w} & 0 & 0 & 0 & W_{u}^{e} & R_{u}^{e} \\
0 & 0 & 0 & I_{\sharp C^{h}} & 0 & 0 & W_{C^{h}}^{e} & R_{C^{h}}^{e} \\
0 & 0 & 0 & 0 & I_{\sharp C^{o}} & 0 & W_{C^{o}}^{e} & R_{C^{o}}^{e} \\
0 & 0 & 0 & 0 & 0 & I_{\sharp q} & W_{q}^{e} & R_{q}^{e}
\end{array}\right]\left[\begin{array}{c}
\delta f_{c^{h}}^{c^{o}} \\
\delta \tau \\
\delta u \\
\delta C_{a c^{h}}^{c^{o}} \\
\delta C_{a c^{o}}^{c^{o}} \\
\delta q \\
\delta w_{e}^{b} \\
\delta q_{r}
\end{array}\right]=0 .
$$

The blocks composing (122), expressed as function of the terms in (123) and (124), have the form

$$
\begin{gathered}
W_{f}^{e}=W_{f}^{f}+R_{f}^{f}\left(K_{q}-R_{\tau}^{f}\right)^{-1} W_{\tau}^{f}, \\
W_{\tau}^{e}=W_{\tau}^{f}+R_{\tau}^{f}\left(K_{q}-R_{\tau}^{f}\right)^{-1} W_{\tau}^{f}, \\
W_{u}^{e}=W_{u}^{f}+R_{u}^{f}\left(K_{q}-R_{\tau}^{f}\right)^{-1} W_{\tau}^{f}, \\
W_{C^{h}}^{e}=R_{C^{h}}^{f}\left(K_{q}-R_{\tau}^{f}\right)^{-1} W_{\tau}^{f}, \\
W_{C^{o}}^{e}=W_{C^{o}}^{f}+R_{C^{o}}^{f}\left(K_{q}-R_{\tau}^{f}\right)^{-1} W_{\tau}^{f}, \\
W_{q}^{e}=-\left(K_{q}-R_{\tau}^{f}\right)^{-1} W_{\tau}^{f}, \\
R_{f}^{e}=R_{f}^{f}\left(K_{q}-R_{\tau}^{f}\right)^{-1} K_{q}, \\
R_{\tau}^{e}=R_{\tau}^{f}\left(K_{q}-R_{\tau}^{f}\right)^{-1} K_{q}, \\
R_{u}^{e}=R_{u}^{f}\left(K_{q}-R_{\tau}^{f}\right)^{-1} K_{q}, \\
R_{C^{h}}^{e}=R_{C^{h}}^{f}\left(K_{q}-R_{\tau}^{f}\right)^{-1} K_{q}, \\
R_{C^{o}}^{e}=R_{C^{o}}^{f}\left(K_{q}-R_{\tau}^{f}\right)^{-1} K_{q}, \\
R_{q}^{e}=-\left(K_{q}-R_{\tau}^{f}\right)^{-1} K_{q} .
\end{gathered}
$$

D.3 Fundamental Grasp Matrix for a Synergistic Underactuated Hand Grasping an Object Using the result in (122), the Fundamental Grasp Equation in (43) can be rewritten as

$$
\left[\begin{array}{ccccccccccc}
I_{\sharp f} & 0 & 0 & 0 & 0 & 0 & R_{f}^{f} & 0 & 0 & W_{f}^{f} & 0 \\
0 & I_{\sharp \tau} & 0 & 0 & 0 & 0 & R_{\tau}^{f} & 0 & 0 & W_{\tau}^{f} & 0 \\
0 & -\bar{S}^{T} & I_{\sharp \eta} & 0 & 0 & 0 & 0 & 0 & -\bar{\Sigma} & 0 & 0 \\
0 & 0 & 0 & I_{\sharp u} & 0 & 0 & R_{u}^{f} & 0 & 0 & W_{u}^{f} & 0 \\
0 & 0 & 0 & 0 & I_{\sharp C^{h}} & 0 & R_{C^{h}}^{f} & 0 & 0 & 0 & 0 \\
0 & 0 & 0 & 0 & 0 & I_{\sharp C^{o}} & R_{C^{o}}^{f} & 0 & 0 & W_{C^{o}}^{f} & 0 \\
0 & I_{\sharp \tau} & 0 & 0 & 0 & 0 & K_{q} & -K_{q} & 0 & 0 & 0 \\
0 & 0 & 0 & 0 & 0 & 0 & 0 & I_{\sharp q_{r}} & -\bar{S} & 0 & 0 \\
0 & 0 & I_{\sharp \sigma} & 0 & 0 & 0 & 0 & 0 & K_{\sigma} & 0 & -K_{\sigma}
\end{array}\right]\left[\begin{array}{c}
\delta f_{c^{h}}^{c^{o}} \\
\delta \tau \\
\delta \eta \\
\delta u \\
\delta C_{a c^{h}}^{c^{o}} \\
\delta C_{a c^{o}}^{c^{o}} \\
\delta q \\
\delta q_{r} \\
\delta \sigma \\
\delta w_{e}^{b} \\
\delta \sigma_{r}
\end{array}\right]=0,
$$


Applying GEROME-B to (129), we arrive to the formulation seen in (44). For later use, we define the matrices

$$
\begin{aligned}
& H=\left(K_{q}-R_{\tau}^{f}\right)^{-1}, \\
& L=\left(K_{\sigma}+\bar{\Sigma}-\bar{S}^{T} R_{\tau}^{f} H K_{q} \bar{S}\right)^{-1}, \\
& T=\bar{S}^{T}\left(I+R_{\tau}^{f} H\right) W_{\tau}^{f} .
\end{aligned}
$$

With these simplifications, the matrices composing (44) can be expressed as follows

$$
\begin{aligned}
& W_{f}^{s}=W_{f}^{f}+R_{f}^{f} H\left(W_{\tau}^{f}+K_{q} \bar{S} L T\right) \\
& W_{\tau}^{s}=W_{\tau}^{f}+R_{\tau}^{f} H\left(W_{\tau}^{f}+K_{q} \bar{S} L T\right) \\
& W_{\eta}^{s}=\bar{S}^{T} W_{\tau}^{f}+\bar{\Sigma} L T+\bar{S}^{T} R_{\tau}^{f} H\left(W_{\tau}^{f}+K_{q} \bar{S} L T\right) \\
& W_{u}^{s}=W_{u}^{f}+R_{u}^{f} H\left(W_{\tau}^{f}-K_{q} \bar{S} L T\right) \\
& W_{C^{h}}^{s}=R_{C^{h}}^{f} H\left(W_{\tau}^{f}+K_{q} \bar{S} L T\right) \\
& W_{C^{o}}^{s}=W_{C^{o}}^{f}+R_{C^{o}}^{f} H\left(W_{\tau}^{f}+K_{q} \bar{S} L T\right) \\
& W_{q}^{s}=-H\left(W_{\tau}^{f}+K_{q} \bar{S} L T\right) \\
& W_{q_{r}}^{s}=-\bar{S}_{T}{ }^{s}, \\
& W_{\sigma}^{s}=-L T \\
& R_{f}^{s}=R_{f}^{f} H K_{q} \bar{S} L K_{\sigma}, \\
& R_{\tau}^{s}=R_{\tau}^{f} H K_{q} \bar{S} L K_{\sigma}, \\
& R_{\eta}^{s}=\left(\bar{S}^{T} R_{\tau}^{f} H K_{q} \bar{S}-\bar{\Sigma}\right) L K_{\sigma} \\
& R_{u}^{s}=R_{u}^{f} H K_{q} \bar{S} L K_{\sigma}, \\
& R_{C^{h}}^{s}=R_{C^{h}}^{f} H K_{q} \bar{S} L K_{\sigma} \\
& R_{C^{o}}^{s}=R_{C^{o}}^{f} H K_{q} \bar{S} L K_{\sigma} \\
& R_{q}^{s}=-H K_{q} \bar{S} L K_{\sigma}, \\
& R_{q_{r}}^{s}=-\bar{S} L K_{\sigma}, \\
& R_{\sigma}^{s}=-L K_{\sigma} .
\end{aligned}
$$

\section{References}

[Angelaki and Soechting, 1993] Angelaki, D. E. and Soechting, J. F. (1993). Non-uniform temporal scaling of hand and finger kinematics during typing. Experimental Brain Research, 92(2):319 - 329.

[Bicchi, 1994] Bicchi, A. (1994). On the problem of decomposing grasp and manipulation forces in multiple whole-limb manipulation. Int. Journal of Robotics and Autonomous Systems, 13:127-147.

[Bicchi, 2000] Bicchi, A. (2000). Hands for dextrous manipulation and powerful grasping: a difficult road towards simplicity. IEEE Transactions on Robotics and Automation, 6:652-662.

[Bicchi et al., 1995] Bicchi, A., Melchiorri, C., and Balluchi, D. (1995). On the mobility and manipulability of general multiple limb robotic systems. IEEE Trans. Robotics and Automation, 11(2):215-228.

[Birglen et al., 2008] Birglen, L., Laliberté, T., and Gosselin, C. (2008). Underactuated robotic hands, volume 40 of Springer Tracts in Advanced Robotics. Springer Verlag. 
[Brown and Asada, 2007] Brown, C. Y. and Asada, H. H. (2007). Inter-Finger Coordination and Postural Synergies in Robot Hands via Mechanical Implementation of Principal Components Analysis. In 2007 IEEE/RSJ International Conference on Intelligent Robots and System, pages 2877-2882.

[Castellini and van der Smagt, 2013] Castellini, C. and van der Smagt, P. (2013). Evidence of muscle synergies during human grasping. Biological Cybernetics, 107(2):233-245.

[Catalano et al., 2012] Catalano, M. G., Grioli, G., Serio, A., Farnioli, E., Piazza, C., and Bicchi, A. (2012). Adaptive synergies for a humanoid robot hand. In IEEE-RAS International Conference on Humanoid Robots, Osaka, Japan.

[Ciocarlie et al., 2007] Ciocarlie, M., Goldfeder, C., and Allen, P. (2007). Dexterous grasping via eigengrasps: A low-dimensional approach to a high-complexity problem. In Proceedings of the Robotics: Science \& Systems 2007 Workshop-Sensing and Adapting to the Real World, Electronically published.

[Cojocaru et al., 2005] Cojocaru, A., Bruce, J. W., and Murty, R. (2005). An Introduction to Sieve Methods and Their Applications. Cambridge Univ. Press, Cambridge.

[Davidson and Hunt, 2004] Davidson, J. and Hunt, K. (2004). Robots and Screw Theory: Applications of Kinematics and Statics to Robotics. Oxford University Press on Demand.

[Fish and Soechting, 1992] Fish, J. and Soechting, J. F. (1992). Synergistic finger movements in a skilled motor task. Experimental Brain Research, 91(2):327 - 334.

[Gabiccini et al., 2011] Gabiccini, M., Bicchi, A., Prattichizzo, D., and Malvezzi, M. (2011). On the role of hand synergies in the optimal choice of grasping forces. Autonomous Robots [special issue on RSS2010], 31(2 - 3):235 - 252.

[Gabiccini et al., 2012] Gabiccini, M., Farnioli, E., and Bicchi, A. (2012). Grasp and manipulation analysis for synergistic underactuated hands under general loading conditions. In International Conference of Robotics and Automation - ICRA 2012, pages 2836 - 2842, Saint Paul, MN, USA.

[Grebenstein et al., 2012] Grebenstein, M., Chalon, M., Friedl, W., Haddadin, S., Wimbck, T., Hirzinger, G., and Siegwart, R. (2012). The hand of the dlr hand arm system: Designed for interaction. I. J. Robotic Res., 31(13):1531-1555.

[Grioli et al., 2012] Grioli, G., Catalano, M., Silvestro, E., Tono, S., and Bicchi, A. (2012). Adaptive synergies: an approach to the design of under-actuated robotic hands. In IROS/RSJ International Conference on Intelligent Robots and Systems, pages 1251-1256. IEEE.

[Hall, 2003] Hall, B. (2003). Lie Groups, Lie Algebras, and Representations: An Elementary Introduction. Graduate Texts in Mathematics. Springer.

[Jacobsen et al., 1984] Jacobsen, S., Wood, J., Knutt, D., and Biggers, K. (1984). The Utah/MIT dextrous hand: work in progress. The International Journal of Robotics Research, 3(4):21-50.

[Latash et al., 2005] Latash, M. L., Krishnamoorthy, V., Scholz, J. P., and Zatsiorsky, V. M. (2005). Postural Synergies and their Development. Neural plasticity, 12(2-3):119-30; discussion 263-72.

[Lovchik and Diftler, 1999] Lovchik, C. and Diftler, M. (1999). The robonaut hand: a dexterous robot hand for space. In Robotics and Automation, 1999. Proceedings. 1999 IEEE International Conference on, volume 2, pages 907-912 vol.2.

[Meyer, 2000] Meyer, C. D. (2000). Matrix analysis and applied linear algebra. Society for Industrial and Applied Mathematics, Philadelphia, PA, USA.

[Murray et al., 1994] Murray, R., Li, Z., and Sastry, S. (1994). A Mathematical Introduction to Robotic Manipulation. CRC Press INC. 
[Prattichizzo et al., 2011] Prattichizzo, D., Malvezzi, M., and Bicchi, A. (2011). On motion and force controllability of grasping hands with postural synergies. In Robotics: Science and Systems VI, pages 49-56, Zaragoza, Spain. The MIT Press.

[Santello et al., 2013] Santello, M., Baud-Bovy, G., and Jörntell, H. (2013). Neural bases of hand synergies. Frontiers in computational neuroscience, 7:23.

[Santello et al., 1998] Santello, M., Flanders, M., and Soechting, J. (1998). Postural hand synergies for tool use. The Journal of Neuroscience, 18(23):10105-10115.

[Sciavicco and Siciliano, 2000] Sciavicco, L. and Siciliano, B. (2000). Modelling and control of robot manipulators. Springer.

[Selig, 2005] Selig, J. M. (2005.). Geometric Fundamentals of Robotics. Monographs in Computer Science. Springer New York, NY, second edition.

[Soechting and Flanders, 1997] Soechting, J. F. and Flanders, M. (1997). Flexibility and repeatability of finger movements during typing: Analysis of multiple degrees of freedom. Journal of Computational Neuroscience, 4(1):29-46.

[Shadow Robot Company Ltd., 2009] Shadow Robot Company Ltd. (2009). Shadow hand. Retrieved from Shadowhand: http://shadowhand.com.

[Thakur et al., 2008] Thakur, P. H., Bastian, A. J., and Hsiao, S. S. (2008). Multidigit Movement Synergies of the Human Hand in an Unconstrained Haptic Exploration Task. Journal of Neuroscience, 28(6):1271-1281. 